\title{
Sexually dimorphic DNA-methylation in cardiometabolic health: A systematic review
}

\author{
Eralda Asllanaj $^{\mathrm{a}, \mathrm{b}, *}$, Xiaofang Zhang ${ }^{\mathrm{a}, 1}$, Carolina Ochoa Rosales ${ }^{\mathrm{a}, \mathrm{c}, 1}$, Jana Nano ${ }^{\mathrm{d}, \mathrm{e}}$, \\ Wichor M. Bramer ${ }^{f}$, Eliana Portilla-Fernandez ${ }^{\mathrm{a}, \mathrm{g}}$, Kim V.E. Braun ${ }^{\mathrm{a}}$, \\ Valentina Gonzalez-Jaramillo ${ }^{\mathrm{h}}$, Wolfgang Ahrens ${ }^{\mathrm{i}}$, Arfan Ikram ${ }^{\mathrm{a}, \mathrm{j}}$, Mohsen Ghanbari ${ }^{\mathrm{a}}$, \\ Trudy Voortman ${ }^{\mathrm{a}}$, Oscar H. Franco ${ }^{\mathrm{a}, \mathrm{h}}$, Taulant Muka ${ }^{\mathrm{h}, 1}$, Marija Glisic ${ }^{\mathrm{h}, \mathrm{k}, 1}$

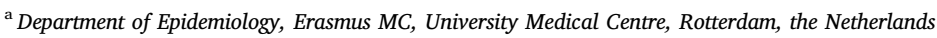 \\ ${ }^{\mathrm{b}}$ Institute for Community Medicine, University Medicine Greifswald, Greifswald, Germany \\ ${ }^{c}$ Centro de Vida Saludable de la Universidad de Concepción, Concepción, Chile \\ ${ }^{\mathrm{d}}$ Institute of Epidemiology, Helmholtz Zentrum München, German Research Centre for Environmental Health, Neuherberg, Germany \\ ${ }^{\mathrm{e}}$ German Centre for Diabetes Research, Neuherberg, Germany \\ ${ }^{\mathrm{f}}$ Medical Library, Erasmus MC, University Medical Centre, Rotterdam, the Netherlands \\ ${ }^{\mathrm{g}}$ Division of Pharmacology, Department of Internal Medicine, Erasmus MC, Rotterdam, the Netherlands \\ ${ }^{\mathrm{h}}$ Institute of Social and Preventive Medicine, University of Bern, Bern, Switzerland \\ ${ }^{\mathrm{i}}$ Leibniz Institute for Prevention Research and Epidemiology-BIPS, Bremen, Germany \\ ${ }^{\mathrm{j}}$ Department of Neurology, Erasmus MC, University Medical Centre, Rotterdam, the Netherlands \\ ${ }^{\mathrm{k}}$ Swiss Paraplegic Research, Nottwil, Switzerland
}

\section{A R T I C L E I N F O}

\section{Keywords:}

DNA methylation

Type 2 diabetes

Coronary disease

Myocardial infarction

Stroke

\begin{abstract}
A B S T R A C T
Sex is a major determinant of cardiometabolic risk. DNA methylation (DNAm), an important epigenetic mechanism that differs between sexes, has been associated with cardiometabolic diseases. Therefore, we aimed to systematically review studies in adults investigating sex-specific associations of DNAm with intermediate cardiometabolic traits and incident cardiovascular disease including stroke, myocardial infarction (MI) and coronary heart disease (CHD). Five bibliographic databases were searched from inception to 15 July 2019 . We selected 35 articles (based on 30 unique studies) from 17,023 references identified, with a total of 14,020 participants of European, North American or Asian ancestry. Four studies reported sex differences between global DNAm and blood lipid levels and stroke risk. In 25 studies that took a genome wide or candidate gene approach, DNAm at 31 gene sites was associated with sex differences in cardiometabolic diseases. The identified genes were PLA2G7, BCL11A, KDM6A, LIPC, ABCG1, PLTP, CETP, ADD1, CNN1B, HOOK2, GFBP-7,PTPN1, GCK, PTX3, ABCG1, GALNT2, CDKN2B, APOE, CTH, GNASAS, INS, PON1, TCN2, CBS, AMT, KDMA6A, FTO, $M A P 3 K 13, C C D C 8, M M P-2$ and $E R-\alpha$. Prioritized pathway connectivity analysis associated these genes with biological pathways such as vitamin B12 metabolism, statin pathway, plasma lipoprotein, plasma lipoprotein assembly, remodeling and clearance and cholesterol metabolism. Our findings suggest that DNAm might be a promising molecular strategy for understanding sex differences in the pathophysiology of cardiometabolic diseases and that future studies should investigate the effects of sex on epigenetic mechanisms in cardiometabolic risk. In addition, we emphasize the gap between the translational potential and the clinical utilization of cardiometabolic epigenetics.
\end{abstract}

\section{Introduction}

Cardiometabolic diseases include cardiovascular diseases (CVD), type 2 diabetes (T2D) and their associated risk factors including components of the metabolic syndrome and obesity [1]. Aging is associated with development of unfavorable cardiometabolic profile which in large contributes to increased incidence of major cardiovascular events and mortality [2]. Intermediate cardiometabolic risk

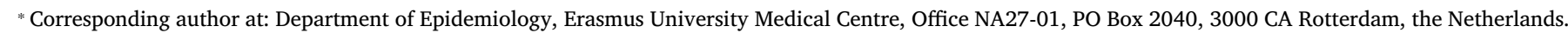
E-mail address: e.asllanaj@erasmusmc.nl (E. Asllanaj).

${ }^{1}$ Denote equal contribution.
} 
factors are unequally distributed among sexes, and sex differences are also described in cardiometabolic diseases prevalence, clinical characteristics and prognosis [3-5]. Generally, before menopause women have better cardiometabolic risk profiles than same aged men; however, this sex advantage gradually disappears with advancing age, particularly after menopause [6]. Mechanisms underlying sex differences in CVD have been extensively studied in the past two decades and signaling pathways including epigenetic modifications of the genome emerged as possible pathways leading to sexual dimorphism in cardiometabolic diseases [7].

Epigenetic modifications comprise dynamic changes in the genome engaged in the modification of important cellular processes such as gene expression, chromosomal stability and genomic imprinting $[8,9]$. DNA methylation (DNAm) is the best understood and most extensively studied epigenetic mechanism in regard to CVD risk $[8,9]$. DNAm refers to the transfer of a methyl group into the $\mathrm{C} 5$ position of the cytosine to form 5-methylcytosine $(5 \mathrm{mC})$ and increases or decreases in genomic $5 \mathrm{mC}$ are referred as DNA hyper- and hypomethylation respectively $[8,9]$. The global DNAm assessed at long-interspersed nuclear element (LINE-1) has been inversely associated with intermediate CVD risk factors and higher risk of metabolic status worsening [10]. Conversely, a higher degree of global DNA methylation measured at Alu repeats or by the LUMA method was associated with the presence of CVD [10]. Also, gene specific hyper- or DNA hypomethylation was associated with changes in gene expression and was shown to affect cardiometabolic risk including atherosclerosis, inflammation, blood pressure, serum lipid and glucose levels, subsequently leading to increased risk of developing T2D, stroke and myocardial infarction [11]. Also, sex-specific differences in DNAm have been found in brain, human pancreatic islets and blood [12,13]. Men in general seem to have lower levels of methylation in their genome as compared to women [14,15], indicating that similarly to sex chromosomes, methylation at the autosomes might be subject to sex differences. Despite this, only a relatively small number of studies in the ample field of cardiometabolic epigenetics stratify according to sex or focus in sex differences. Although a few studies $[11,16,17]$ have summarized the existing literature on this complex topic, they did not focus on epigenetically induced sex differences in CVD, intermediate CVD risk factors or T2D, with the exception of a recent review that focused only on major CVD events [16].

Therefore, we aimed to systematically review the available evidence in human studies exploring the association between sex-specific DNAm and cardiometabolic diseases.

\section{Methods}

\subsection{Data sources and searches}

This review was conducted using a predefined protocol (which was not registered at online platforms) and following a recently published guideline on how to perform systematic reviews [18] and in accordance with PRISMA guidelines [19]. A literature search was done using 5 electronic databases (Medline ALL via Ovid (1946-current), EMBASE (1974-current) via embase.com, Web of Science Core Collection (1900current), Cochrane CENTRAL registry of trials (issue 7 2019) via Wiley and Google Scholar) until 15 July 2019 (date last searched) with the help of an experienced medical information specialist (WMB). Details on the search strategy are provided in Supplemental Table 1. All references were imported in an EndNote library and deduplicated with the algorithm developed by Bramer et al. [20]. Additionally, we searched the reference lists of the included studies and relevant reviews. Two independent reviewers screened the titles and abstracts of all studies identified initially, according to the selection criteria. Full texts were retrieved from studies that satisfied all selection criteria. All disagreements were resolved through consensus or consultation with two other independent reviewers.

\subsection{Study selection}

Observational (cross-sectional, case-control, prospective) studies conducted in adults and investigating the associations of global or genespecific DNAm with cardiometabolic outcomes were selected. Studies were included if they investigated sex-stratified associations between DNAm and intermediate cardio-metabolic traits (blood lipids, glucose, blood pressure, inflammatory markers, atherosclerosis, T2D) and CVD (MI, CHD, stroke). Also, we included studies that reported a significant interaction term with sex but did not stratify by sex in their analysis. Furthermore, studies conducted only in men/women were not included in the current review.

\subsection{Data extraction}

A predesigned electronic data abstraction form was used to extract relevant information. This included questions on study location, percentage of men and women included in the study, participants' age, cardio-metabolic outcome, tissue type, DNAm technique used and general and sex-specific findings. Two authors independently extracted the data and a consensus was reached in case of any inconsistency with involvement of an additional author.

\subsection{Assessing the risk of Bias}

Two independent investigators used the Newcastle-Ottawa Scale [21] to assess the risk of bias of the included observational studies. The Newcastle-Ottawa Scale uses a star system (maximum of nine stars) to evaluate three domains: selection of participants; comparability of study groups; and the ascertainment of outcomes and exposures of interest. Studies that received a score of nine stars were judged to be of low risk of bias; a score of seven or eight stars was medium risk and those that scored six or less were considered at high risk of bias.

\subsection{Pathway connectivity analysis}

To identify biological pathways of the differentially methylated genes previously linked to CVD, we used the CPDB (ConsensusPathDBhuman) tool from the Max Plank Institute for Molecular Genetics [22]. This tool integrates interaction networks in humans (in this study) and includes information on binary and complex protein-protein, genetic, metabolic, signaling, gene regulatory and drug-target interactions, as well as biochemical pathways [22]. Data that explains interactions was derived from 32 public resources.

\section{Results}

\subsection{Search results and study characteristics}

The search strategy identified 17,023 potentially relevant studies; after titles and abstracts were screened 16, 814 references were excluded (Fig. 1). For the remaining 209 references, full-text articles were reviewed, 174 of which were excluded for various reasons outlined in Fig. 1. A total of 35 articles based on 30 unique studies were included in this systematic review including a total of 14,020 non-overlapping participants, of whom approximately $53 \%$ were women. The studies included population with European $(\mathrm{n}=13)$, North American $(\mathrm{n}=3)$ and Asian $(n=14)$ ancestries with age ranging from 32 to 75 years. Due to differences in the epigenetics marks and outcomes investigated, as well as different study designs of the included studies, we were not able to quantitatively pool the estimates from various studies. Therefore we report in this review a detailed descriptive summary of the available published literature. The characteristics of the included studies are described in Table 1. The median Newcastle-Ottawa quality score for the included studies was 7 of 9 possible points. The Table 1 depicts the methodologic quality of all included studies. 


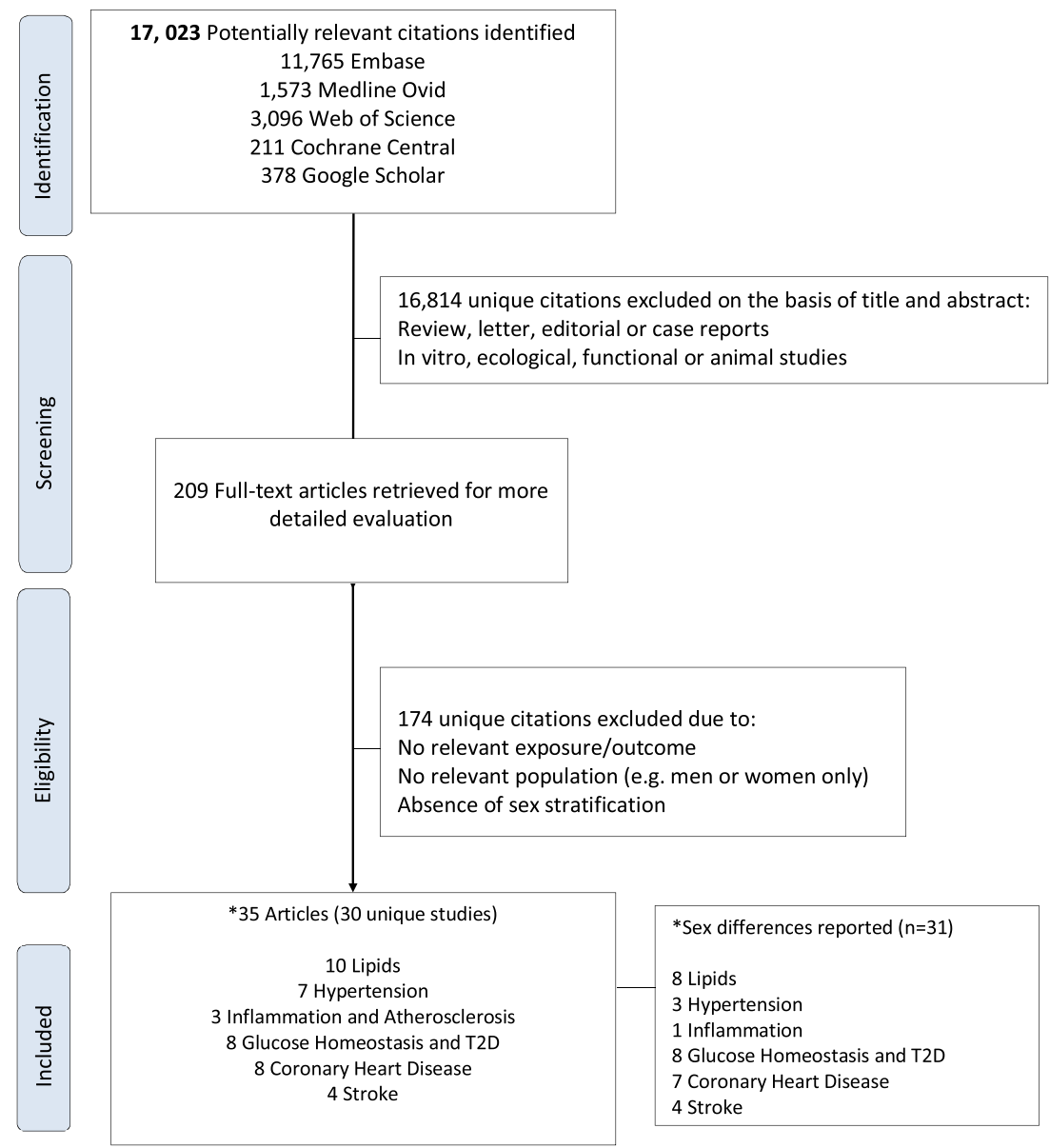

Fig. 1. The flowchart of included studies according to PRISMA guidelines.

*In a specific study more outcomes might have been investigated, therefore, numbers refer to number of articles and not numbers of unique studies'.

\subsection{The Role of Sex-Specific DNAm in Intermediate Cardio-metabolic} Traits

\subsubsection{Blood lipids}

Global DNA methylation is a frequent used marker for epigenetic screening since it captures the DNA methylation also at unknown genetic locations while the results of average DNA methylation correlate with the methylation of some trait-relevant genes [23,24]. Ten articles [14,25-33] investigated sex-specific associations between DNAm and blood lipid concentrations applying global $\mathrm{n}=2$, epi-genome wide $\mathrm{n}=1$ and candidate gene $\mathrm{n}=7$ approaches. In total 2,443 non-overlapping participants 1,174 women and 1,269 men from USA, Canada, Finland, UK and China were included in these studies. The summary of the sex-specific findings is described in Supplemental Fig. 1.

Two cross-sectional studies $[14,25]$ investigating global DNAm and blood lipid levels reported sex differences. In the study conducted by Cash et al., LINE-1 methylation was significantly higher in men than in women, and among the entire sample, lower levels of LINE-1 methylation was associated with higher levels of fasting low-density lipoprotein-cholesterol (LDL) and lower levels of fasting high-density lipoprotein-cholesterol (HDL) [14]. However, when stratifying by sex, the inverse association between global LINE-1 methylation, LDL and HDL remained significant only in men [14]. Malipatil et al., in their study reported that an increase of $10 \%$ in LINE-1 methylation was associated with decreased cholesterol/HDL ratio by $0.4 \mathrm{mmol} / \mathrm{L}$ in the overall sample of men and women. However, when stratifying by sex, the inverse association remained significant only in women [25]. In an EWAS performed by Garcia-Calzon et al., female samples displayed on average higher methylation in the X-chromosome, whereas males presented higher methylation in the autosomes. Further, women showed higher HDL levels, which were associated with higher KDM6A expression and epigenetic differences in human liver [31]. The results were not replicated. Further, the authors integrated DNA regulatory regions and other epigenetic factors for CpGs in the autosomal and Xchromosome based on sex $(\mathrm{q}<0.05)$ for only four liver donors. Particularly, $42 \%$ of the autosomal CpG sites $(13,817 \mathrm{CpGs})$ and $27 \%$ of the X-chromosome sites (2601 CpGs) differentially methylated by sex overlapped with histone marks related to active chromatin and enhancer regions (H3K4me1), whereas $14 \%$ of the autosomal sites (4760 CpGs) and $11 \%$ of the X-chromosome sites overlapped with histone marks related to heterochromatin (H3K27me3) [31].

In three candidate gene-studies PTPN1 [32], PLA2G7 [26] and $B C L 11 A$ [27] DNAm was positively associated with serum lipids in women, but not in men. Another study reported that methylation at $A B C G 1$ was negatively associated with triglycerides in women only [28], whereas methylation at LIPC was negatively associated with triglycerides only in men [27]. This latter study also reported sex-specific associations for total cholesterol: whereas $A B C G 1$ was associated with triglycerides only in women, methylation at this $\mathrm{CpG}$ site was inversely associated with total cholesterol only in men [28]. For some CpG sites there were specific associations with HDL for males; methylation at PLTP [28], CETP [29], and LIPC [28] were negatively associated with HDL in men, but not in women. Moreover, a male-specific association was found between GCK CpG4 methylation at GCK and total cholesterol concentration [33]. However, a single study performed among 739 African Americans in the Genetic Epidemiology Network of Arteriopathy (GENOA) did not find overall or sex-specific significant associations between DNA methylation and lipid levels [30]. 


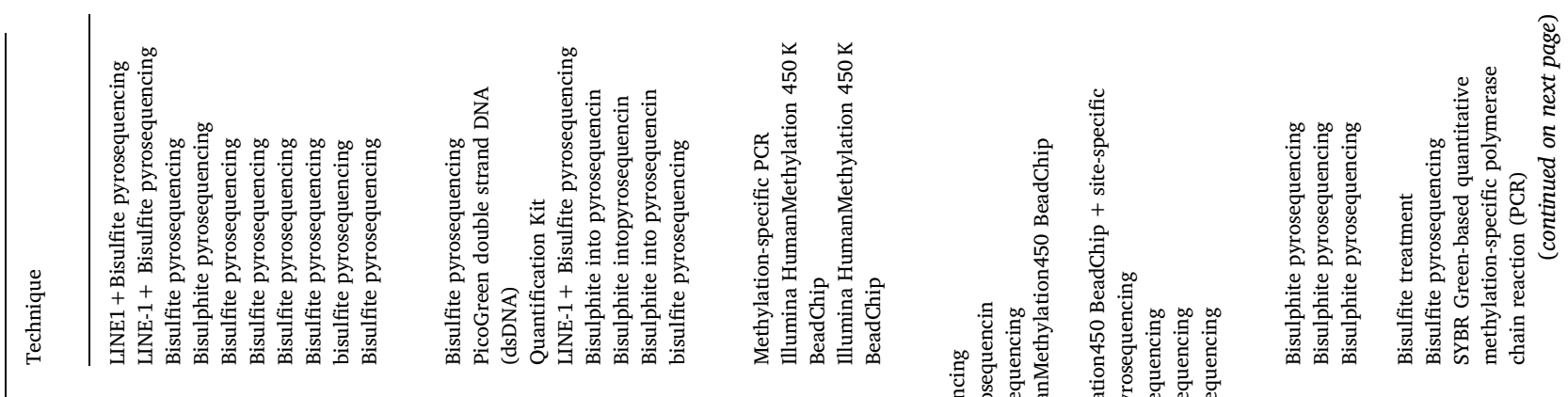

WWIn, II InI In

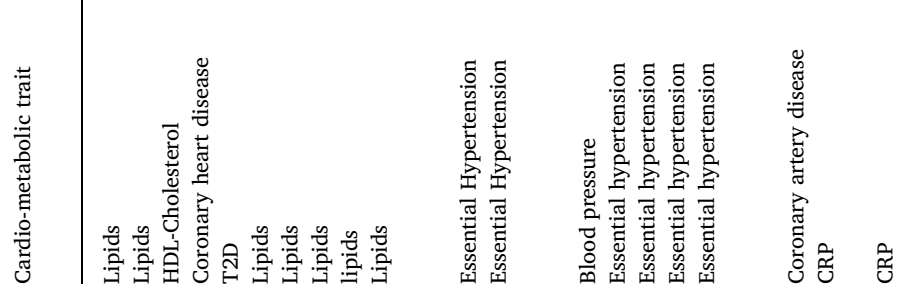

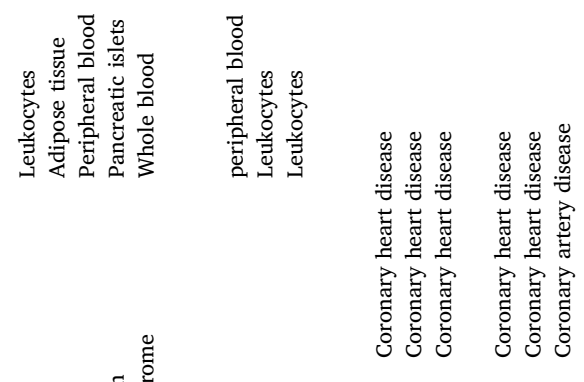

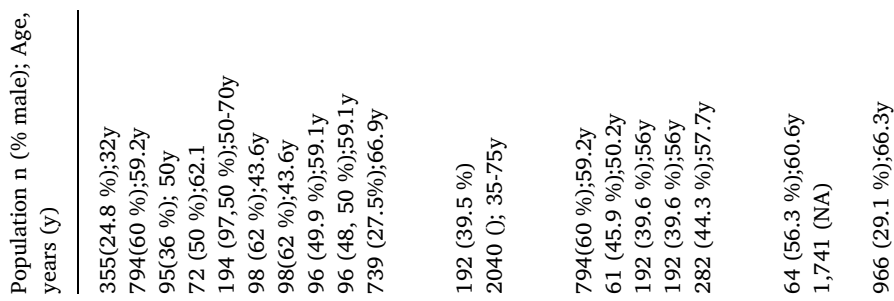

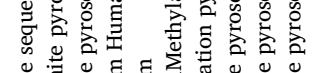

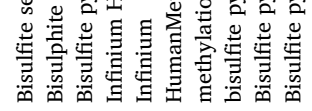

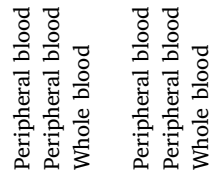

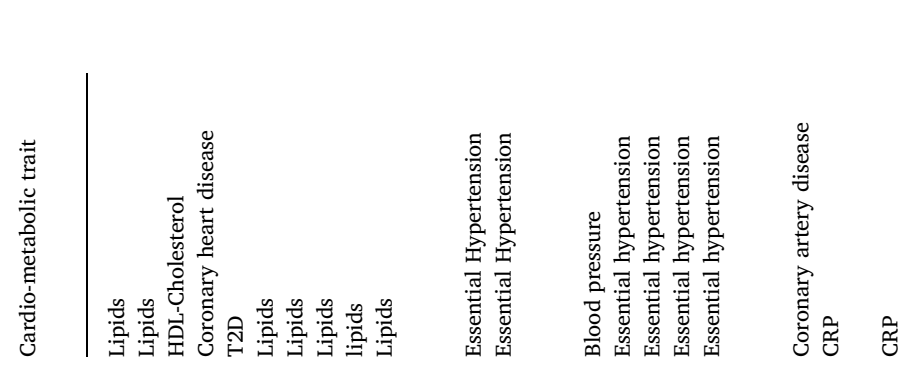

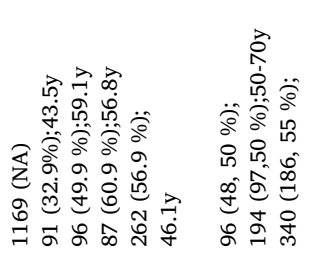

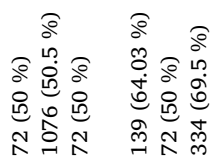

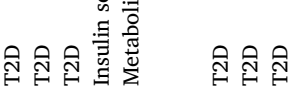

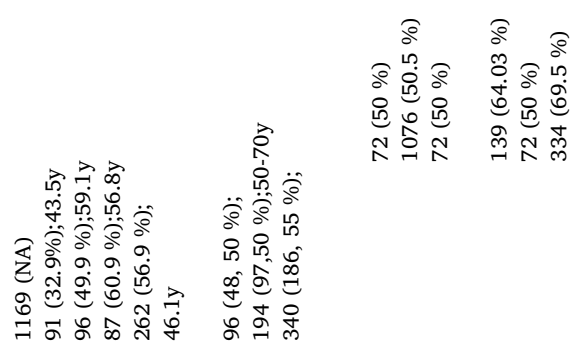

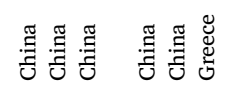

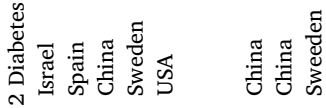

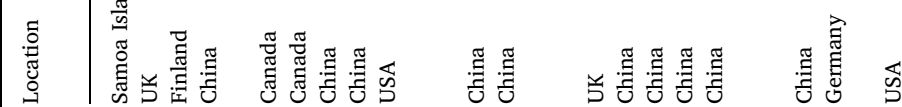




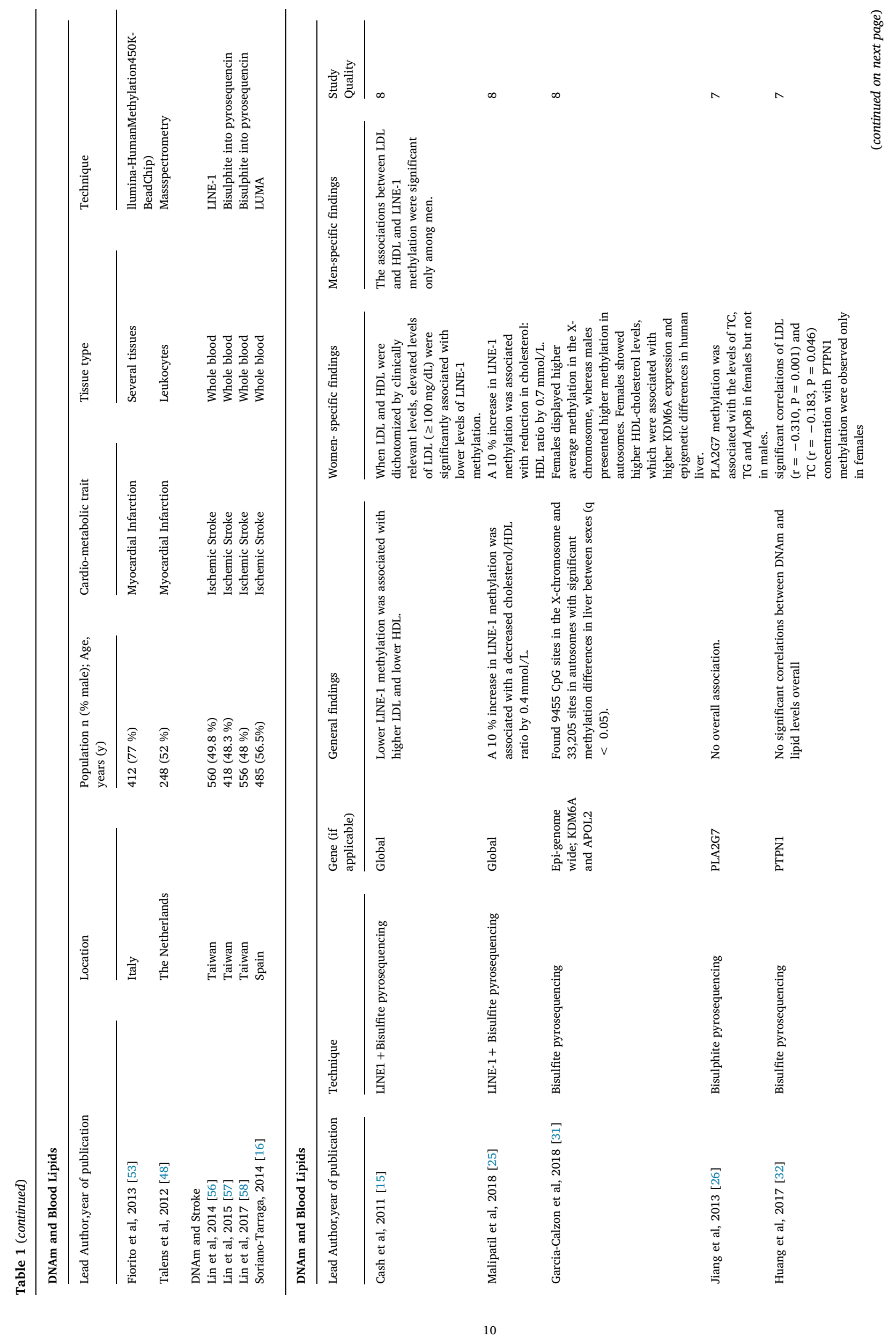




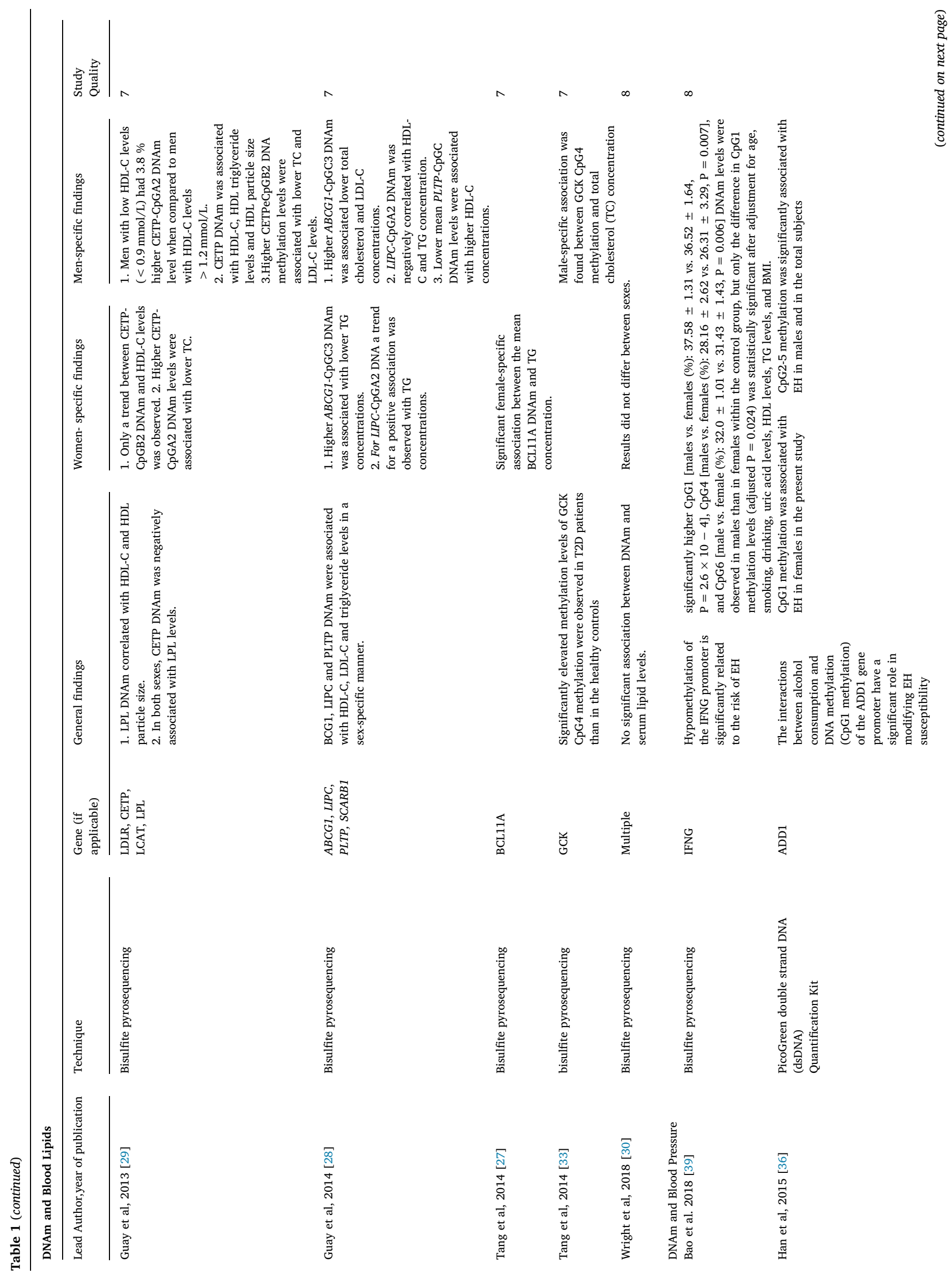




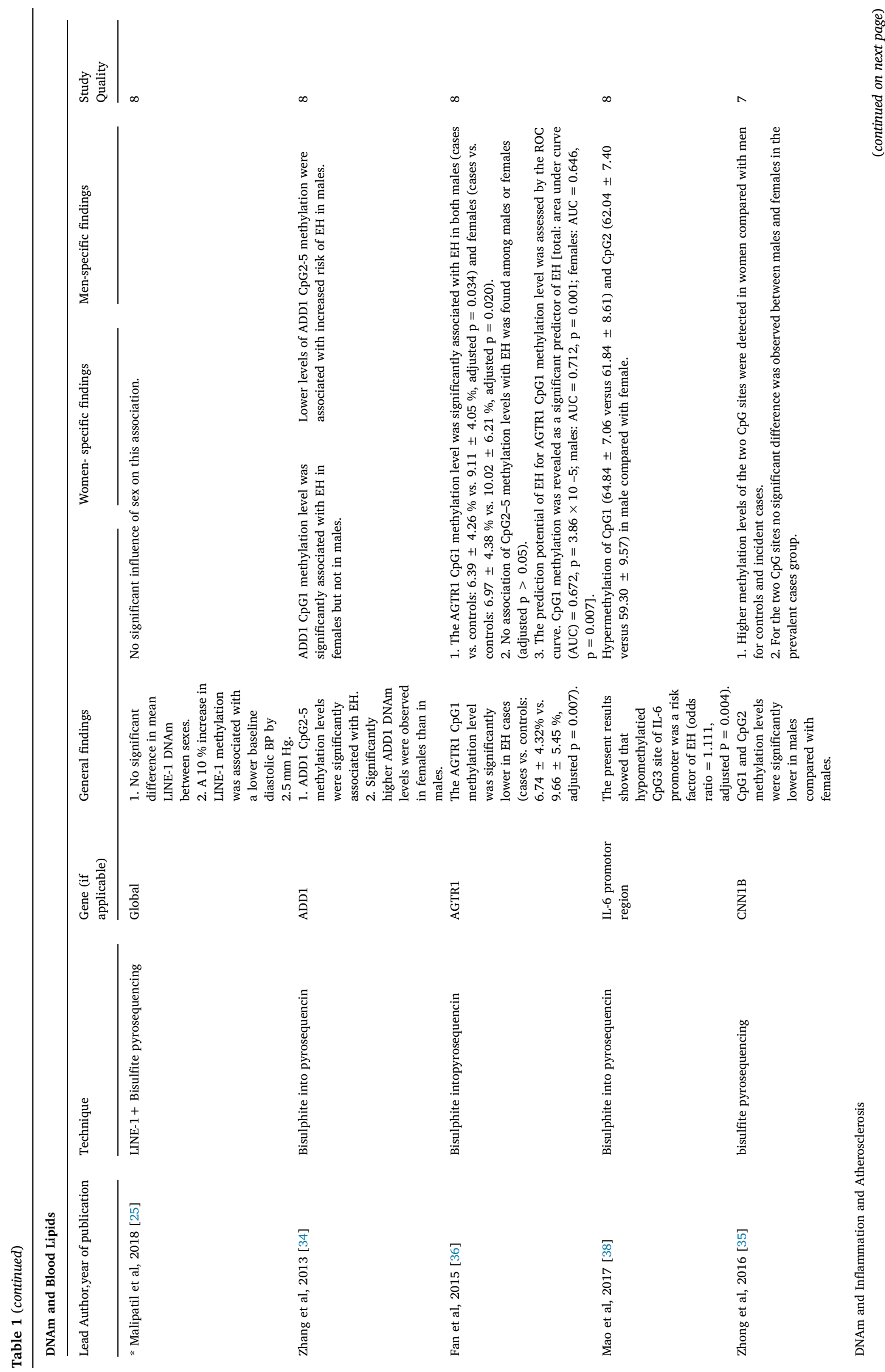




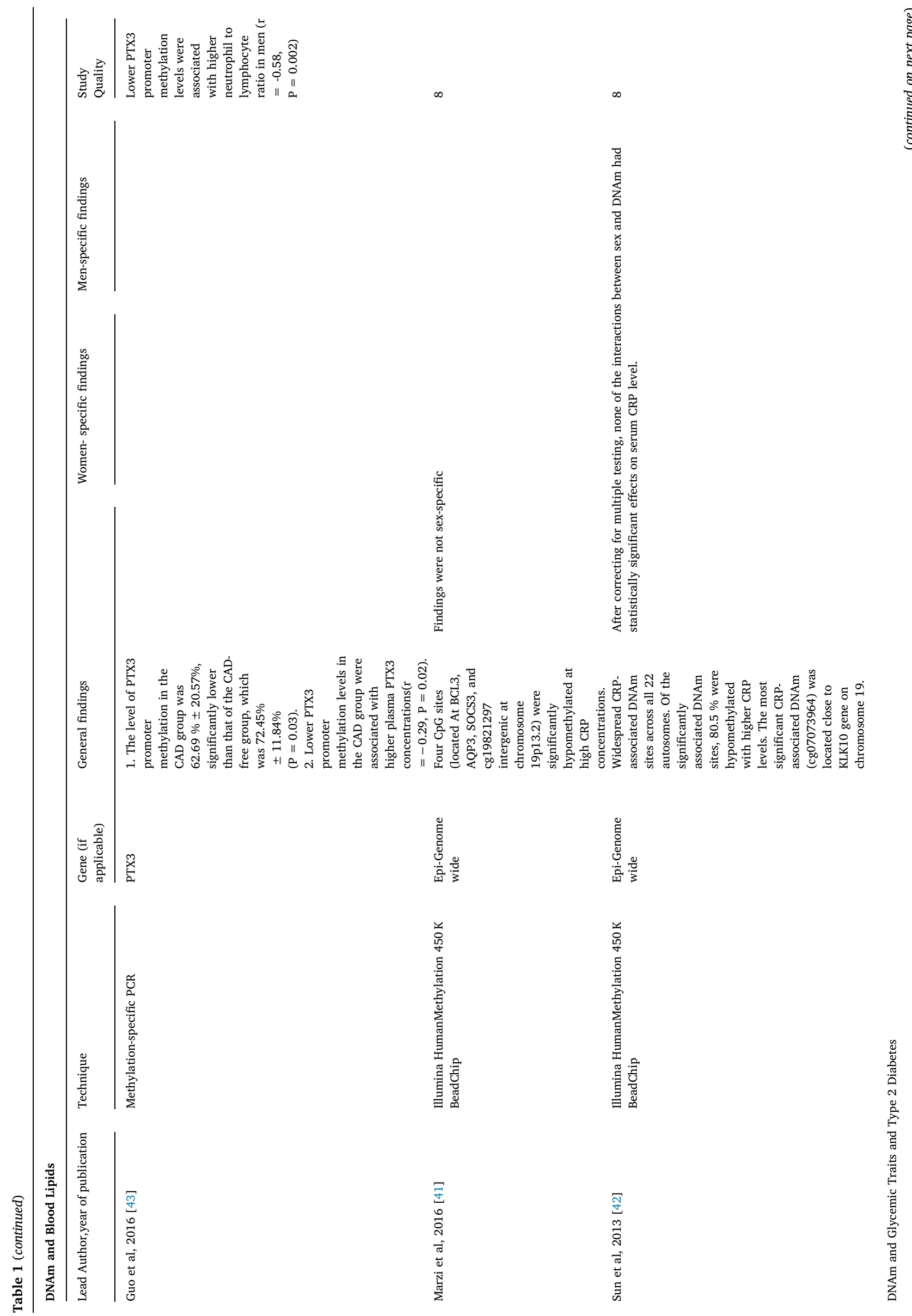




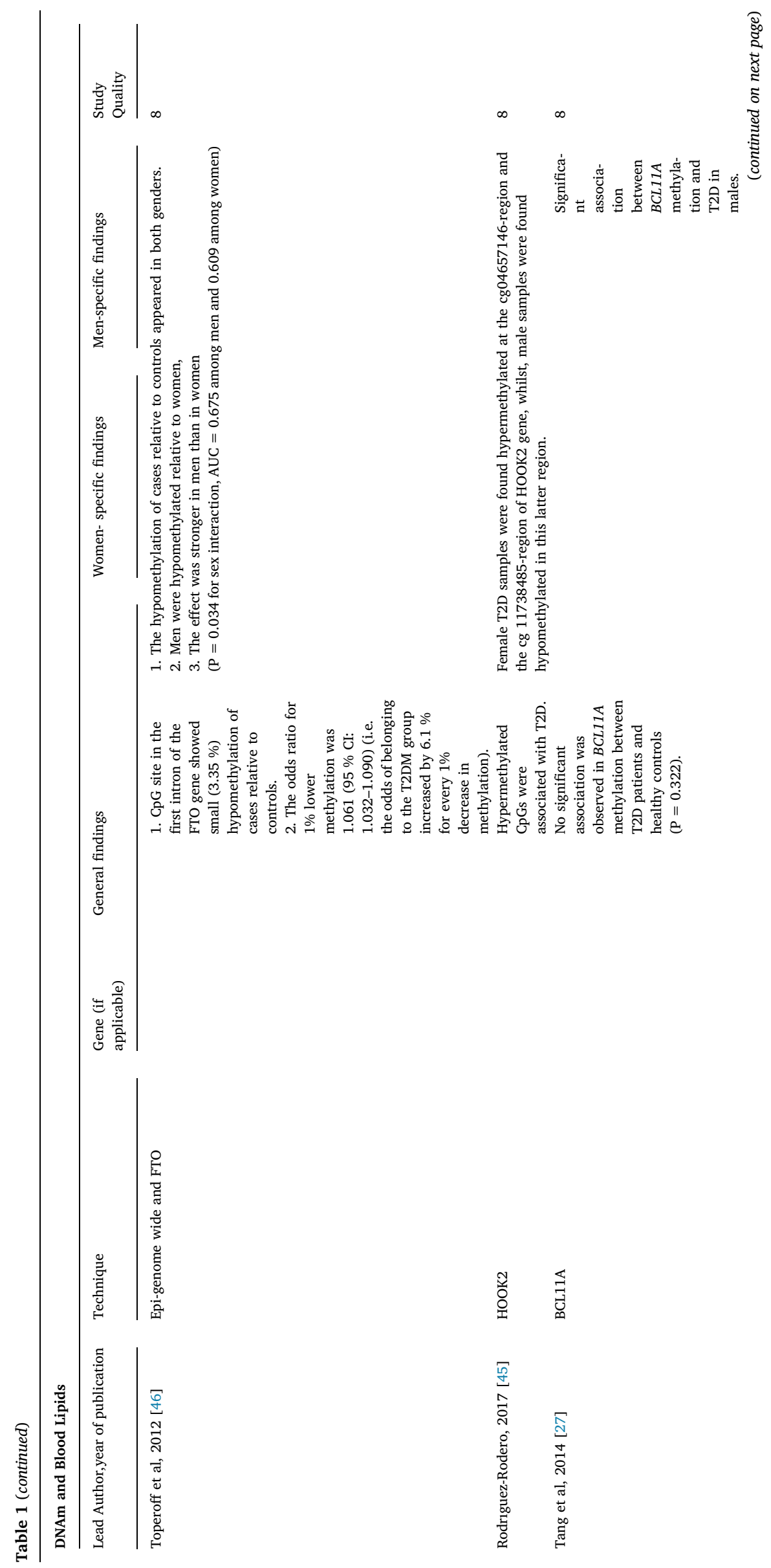




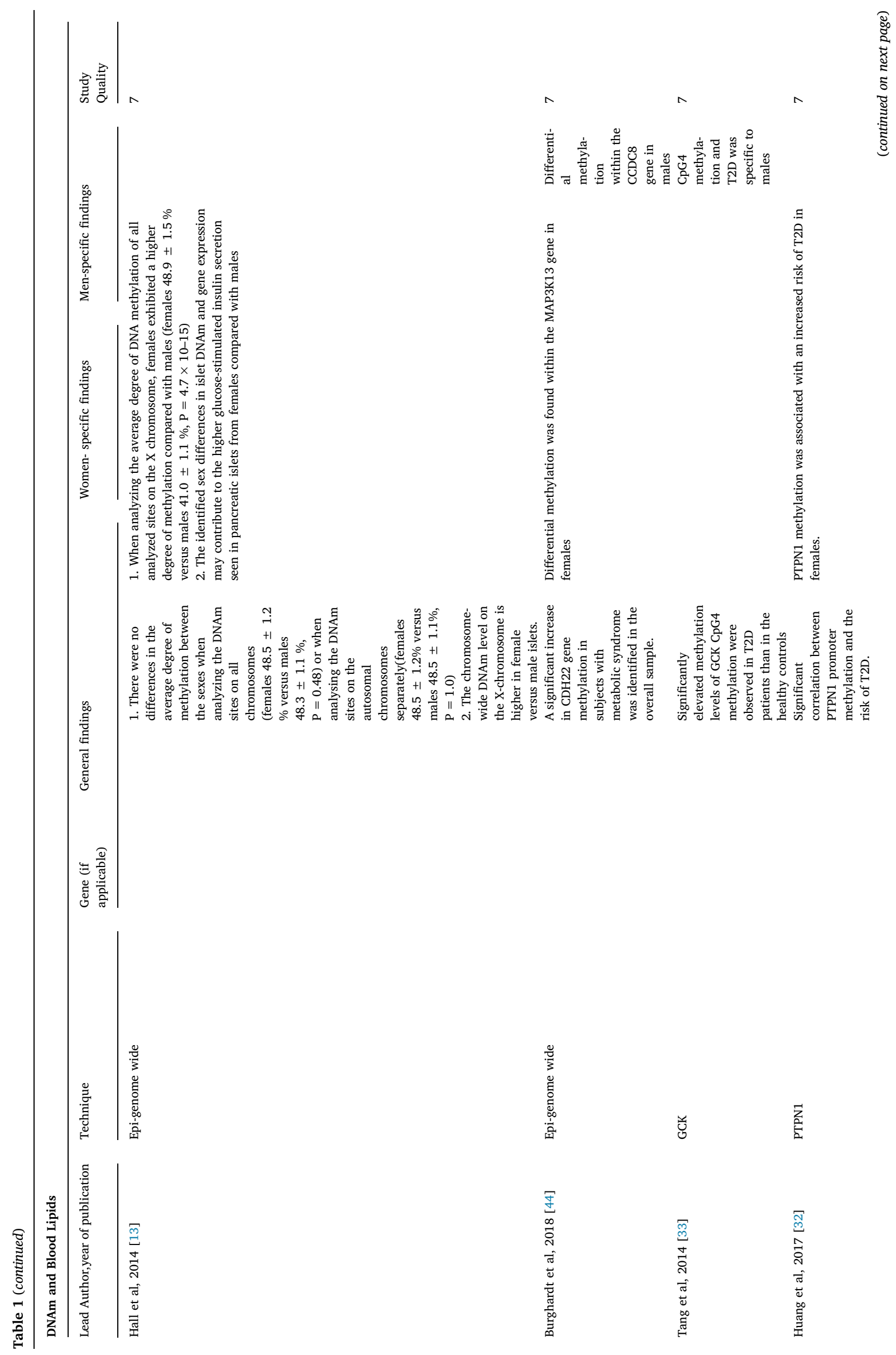




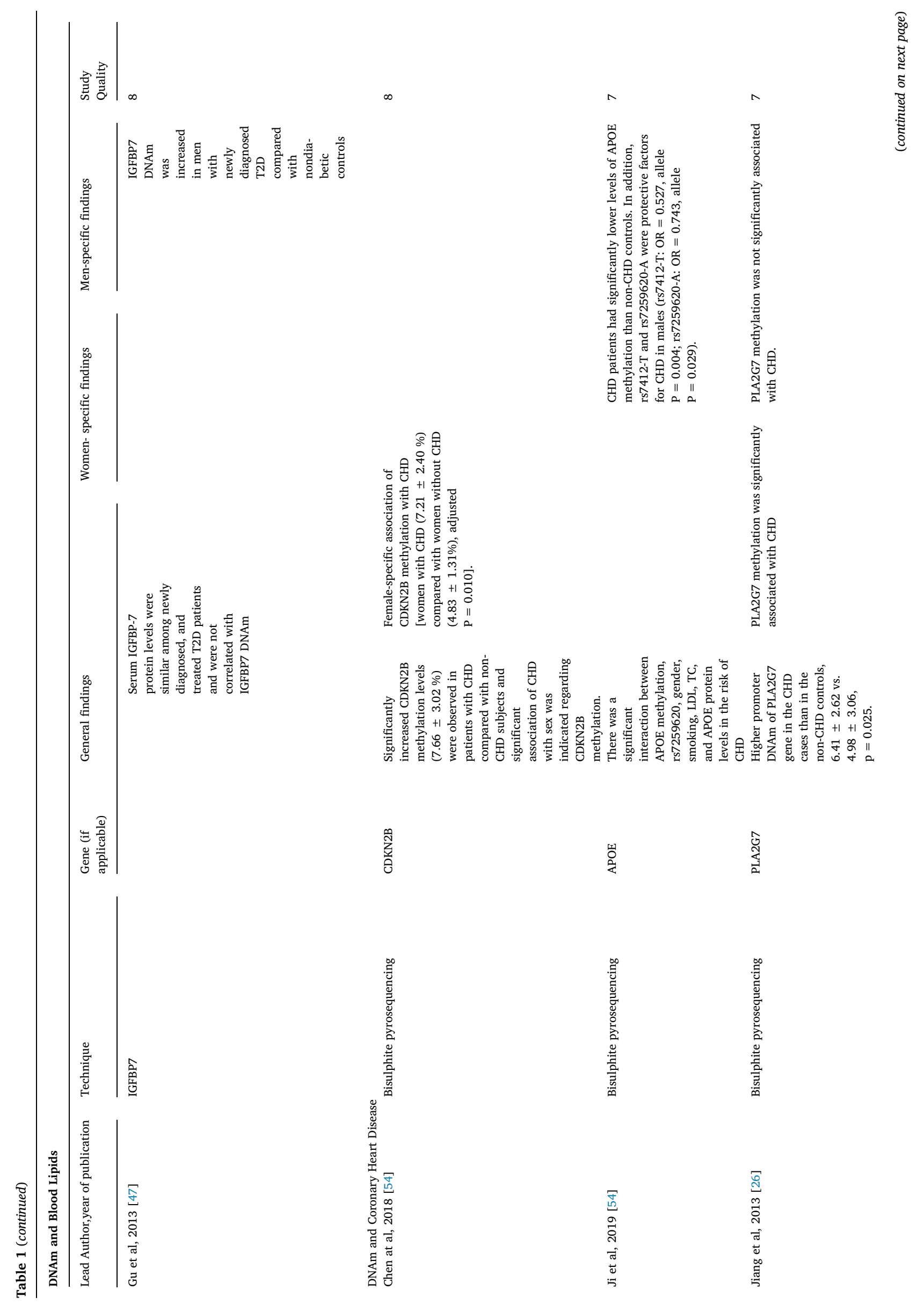




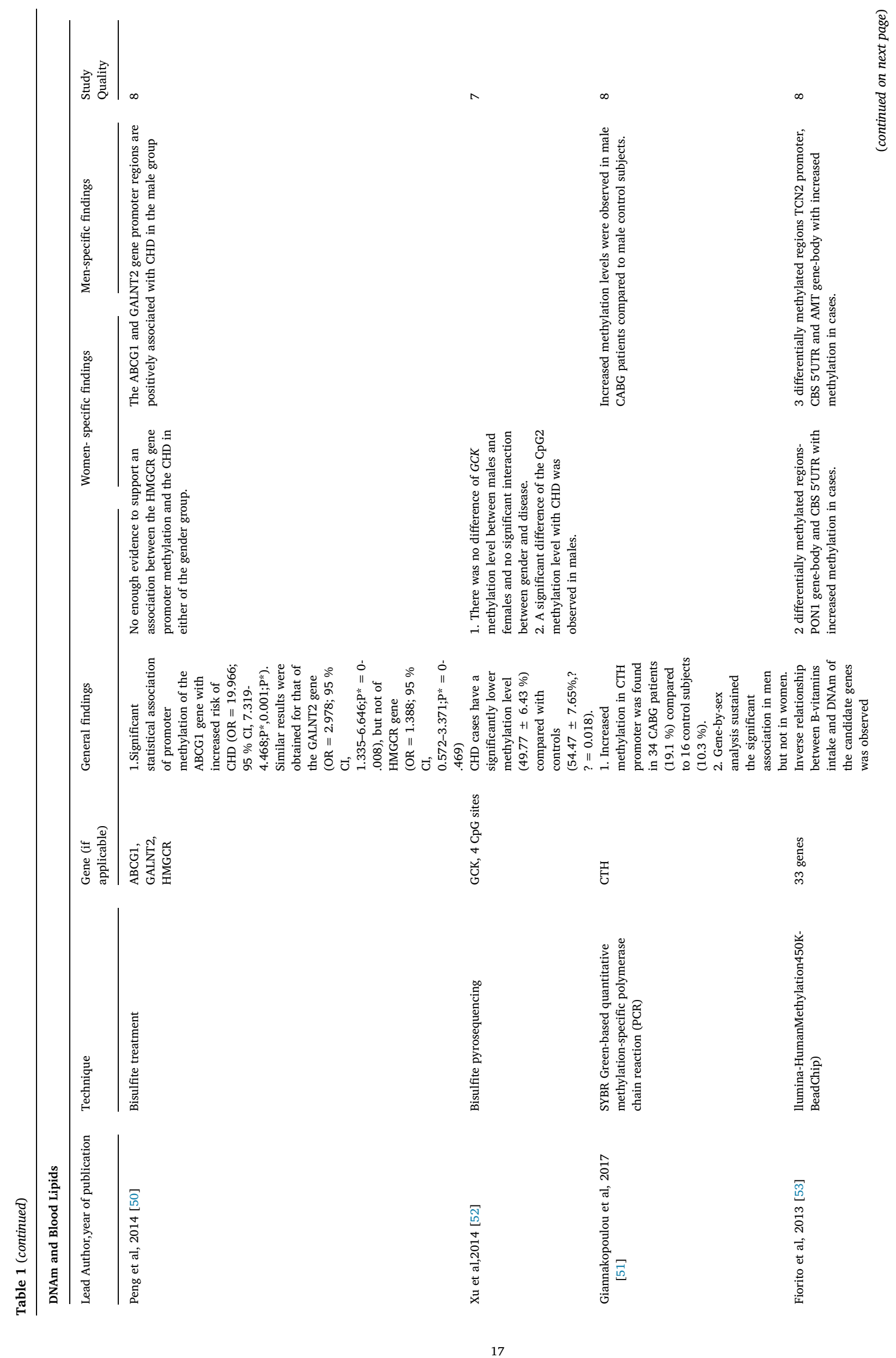




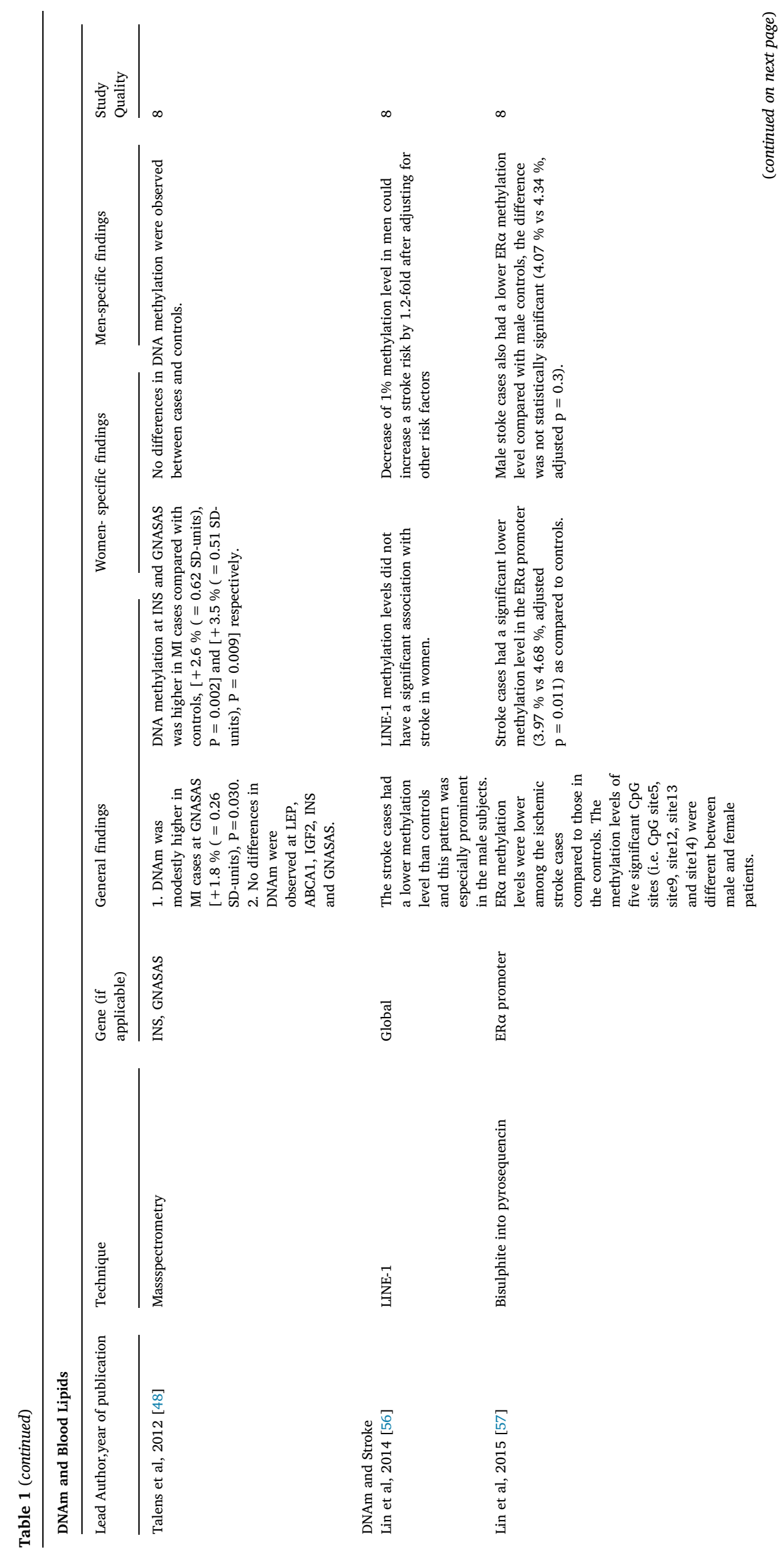




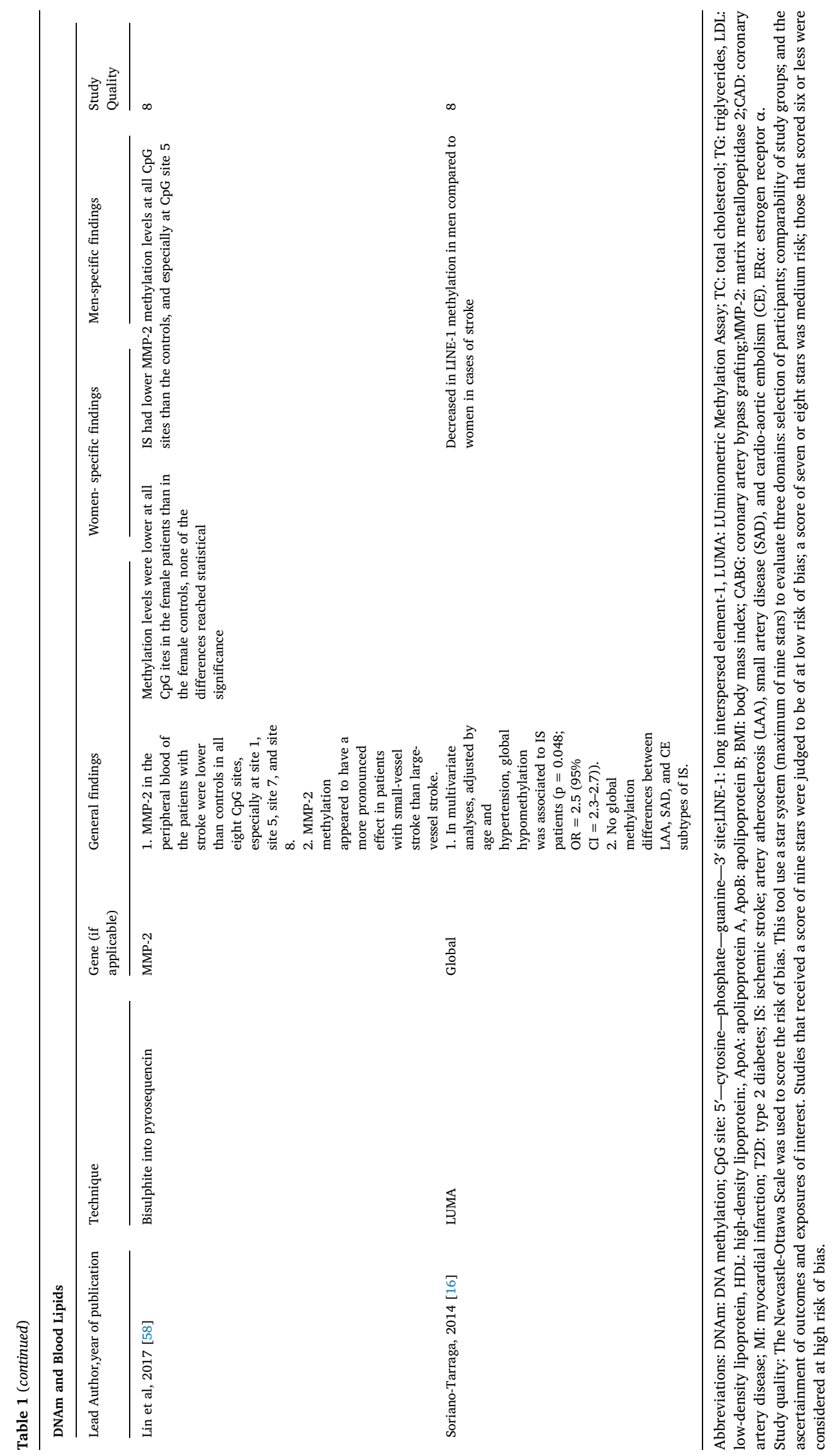




\subsubsection{Blood pressure}

Seven articles [25,34-39] based on five unique studies reported associations between sex-specific DNAm and essential hypertension $(\mathrm{EH})$, while one study investigated the cross-sectional association between DNAm and blood pressure [25]. Among these, five studies investigated candidate gene methylation [34-36,38], while another study investigated epi-genome wide methylation [40] in regard to hypertension. Studies included 3,561 non-overlapping participants 2,029 women and 1,373 men from China and UK. The summary of sex-specific findings is reported in Supplemental Fig. 1.

In the study conducted by Malipatil et al., in the overall sample of men and women, a $10 \%$ increase in LINE- 1 methylation was associated with a $2.5 \mathrm{mmHg}$ lower baseline diastolic blood pressure. The stratified analysis by sex did not show any significant influence of sex on this association [25]. One candidate gene study reported higher methylation levels of the two CpG sites at SCNN18 in women compared with men as controls (CpG1: $\mathrm{t}=-2.283, \mathrm{P}=0.025$; CpG2: $\mathrm{t}=-2.568$, $\mathrm{P}=0.012$ ) and incident $\mathrm{EH}$ cases (CpG1: $\mathrm{t}=-2.694, \mathrm{P}=0.009$; $\mathrm{CpG} 2$ : $t=-2.583, P=0.011$ ) [35]. However, for these two CpG sites no significant difference was observed between males and females in the prevalent cases group (CpG1: $\mathrm{t}=0.409, \mathrm{P}=0.068$; $\mathrm{CpG} 2 \mathrm{t}=0.621$, $P=0.536$ ) [35]. These results indicated a significant association between EH and SCNN1B methylation, which was affected by age, sex and antihypertensive therapy. Similarly, in one other study, higher ADD1 DNAm levels were observed in females as compared to males (CpG1: $\mathrm{P}=0.016$; CpG2-5: $\mathrm{P}=0.021$ ) [34]. Further, the study showed that lower $A D D 1 \mathrm{CpG} 1$ methylation levels were significantly associated with $\mathrm{EH}$ in females (cases versus controls (\%, SD): $10.00 \pm 1.41$ versus $11.36 \pm 3.63$, adjusted $\mathrm{P}=0.042$ ) but not in males (adjusted $\mathrm{P}=0.133$ ). In contrast, lower levels of $A D D 1 \mathrm{CpG} 2-5$ methylation were associated with an increased risk of $\mathrm{EH}$ in males (cases versus controls: $22.48 \%$ versus $31.86 \%$, adjusted $\mathrm{P}=0.008$ ) but not in females [34]. The prediction potential of $\mathrm{EH}$ for $A D D 1 \mathrm{CpG} 1$ and $\mathrm{CpG} 2-5$ methylation levels was assessed by the ROC curves. CpG2-5 methylation was reported as a significant predictor of $\mathrm{EH}$ in males (area under curve (AUC) $=0.855, \mathrm{P}=0.001$ ), while CpG1 methylation showed a trend toward being an $\mathrm{EH}$ indicator in females $(\mathrm{AUC}=0.699, \mathrm{P}=0.054$ ) [34]. In the same population, AGTR1 CpG1 methylation was a significant predictor of EH in both sexes [36] and hypomethylation of CpG3 site at $I L-6$ promotor was significantly associated with EH risk in both, men and women. Further, sex-specific DNAm levels were observed only at CpG1 and CpG2 sites of $I L-6$ promoter (males were hypomethylated as compared to females) [38]. Another study by Han et al., investigated the interactions between alcohol consumption and DNA methylation of the ADD1 gene promoter and its association with $\mathrm{EH}$, involving 2040 cases and controls [36]. The researchers concluded that CpG1 methylation was associated with $\mathrm{EH}$ in females while CpG2-5 methylation was significantly associated with $\mathrm{EH}$ in males, suggesting that these interactions in the $A D D 1$ gene promoter might play a role in modifying EH susceptibility [36]. Finally, Bao et al. reported that hypomethylation of the IFNG promotor region was associated with a higher risk of EH. However, the authors did not observe any sex differences overall, except that in the control group DNAm levels were found to be higher in males when compared to females [39].

\subsubsection{Inflammation and atherosclerosis}

Three articles [41-43], investigated the associations between epigenome wide DNAm $\mathrm{n}=2$, candidate gene methylation and inflammatory markers. Also, we did not identify any study investigating the sex-specific role of DNAm in atherosclerosis. Studies in inflammatory markers included 2,771 non-overlapping participants 713 women and 317 men and one study did not report the number of men and women separately [41]) from Germany, China and USA. None of the epi-genome wide studies reported sex specific associations between global DNAm and inflammatory markers [41,42]. Nevertheless, Guo et al., reported men-specific association between lower PTX3 promoter methylation levels and higher neutrophil to lymphocyte ratio. Also, the level of PTX3 promoter methylation in the coronary artery disease group (mean, SD: $62.69 \% \pm 20.57 \%$ ) was significantly lower than that of the group free of coronary artery disease (mean, SD: 72.45 $\% \pm 11.84 \%$ ), suggesting a role of this gene in developing coronary artery disease [43] (Supplemental Fig. 1).

\subsubsection{Glycemic traits and $T 2 D$}

Eight articles [12,27,32,33,44-47] reported sex-specific associations between DNAm and glycemic traits and T2D. Five studies were candidate gene studies and three were epi-genome wide studies. Among them, six studies focused on DNAm and T2D, one investigated the association between DNAm and metabolic syndrome and another investigated insulin metabolism. Studies included 2,239 non-overlapping participants 353 women and 554 men, with one study not specifying the number of men and women [46]) from Israel, Spain, Sweden, China and USA. Summary of the sex-specific findings is shown in Supplemental Fig. 1.

In a case-control study including 1,169 individuals, individual methylation levels at the FTO gene showed that CpG sites in the first intron were slightly ( $3.35 \%$ ) hypomethylated in T2D cases relative to controls [46]. The odds of developing T2D increased by $6.1 \%$ for every $1 \%$ decrease in DNAm. Men were hypomethylated relatively to women and the effect of DNAm was stronger in males compared to females $(\mathrm{P}=0.034$ for sex interaction, AUC $=0.675$ among men and 0.609 among women) [46]. Also, in another case-control study association between PTPN1 promoter methylation and the risk of T2D was observed in the overall population and in females [32].

In the study by Burghardt et al., a significant increase in $\mathrm{CDH} 22$ gene methylation in subjects with metabolic syndrome was identified in the overall sample [44]. However, when investigating males and females separately; differential methylation levels were observed within the MAP3K13 gene in females and the CCDC8 gene in males with metabolic syndrome. In the validation sample a significant difference in methylation was again observed for the $C D H 22$ and MAP3K13 genes, but not for $C C D C 8$ gene [44]. Another study investigated the impact of sex on the genome-wide DNAm pattern in human pancreatic islets from 53 males and 34 females, and relate the methylome to changes in expression and insulin secretion [12]. The study identified both chromosome-wide and site-specific sex differences in DNA methylation at the $\mathrm{X}$ chromosome of human pancreatic islets. However, the autosomal chromosomes showed differences in DNA methylation only on the level of individual CpG sites between sexes. Importantly, they found higher insulin secretion in pancreatic islets from females compared with males, as well as sex differences in gene expression [12]. Additionally, the authors did not find any difference in $\beta$-cell number between females and males. This could suggest that the DNA methylation differences seen between males and females might not be due to altered $\beta$-cell composition in the islets [12].

In a case-control study conducted by Rodriguez-Rodero et al. [45], hypermethylation at $\mathrm{CpG}$ sites annotated to the HOOK2 gene was associated with the presence of T2D. Interestingly, when these results were analyzed by sex, female T2D samples were found hypermethylated at the cg04657146-region and the cg11738485-region of the HOOK2 gene, whilst male samples were found hypomethylated in this latter region only [45]. Tang et al. reported a significant association only in males when investigating the overall BCL11A methylation in T2D patients [27]. While in another study among the same population, significantly elevated methylation levels of GCK CpG4 were observed in T2D patients than in the healthy controls. Also, this association was characteristic to males only [33]. Further, serum IGFBP-7 protein levels were similar among newly diagnosed and treated T2D patients and were not correlated with IGFBP7 DNAm overall, but solely in males [47]. 


\subsection{The role of sex-specific DNAm in CVD}

\subsubsection{Coronary heart disease}

Eight articles [48-55] investigated the associations between DNAm and CHD and MI. All studies applied a candidate-gene approach and included a total of 2,353 participants 1,010 women and 1,343 men from China, Italy and the Netherlands. The summary of sex-specific findings can be found in Supplemental Fig. 2.

One study reported that a higher DNAm at the imprinted loci of INS and GNASAS was associated with the incidence of MI in women (INS: $+2.5 \%, \mathrm{P}=0.002$; GNASAS: $+4.2 \%, \mathrm{P}=0.001)$ [48]. Hypermethylation at one locus and at both loci was associated with odds ratios (ORs) of 2.8 and 8.6, respectively (Ptrend $=3.0 \times 10-4$ ) while no association was observed among men. Similarly, one study revealed a female-specific significant association between methylation at PLA2G7 promoter and risk of CHD [49]. Another study reported a female specific association of $C D K N 2 B$ methylation with CHD [women with CHD (mean, SD: $7.21 \pm 2.40 \%$ ) compared with women without CHD. In contrast, four studies reported men-specific associations between DNAm of various genes and CHD [43,50,51]. Peng et al., reported significant associations of the methylated promoter of the ABCG1 and GALNT2 genes with an increased risk of CHD overall and among men only [50]. Also, CHD patients had significantly lower levels of APOE methylation than non-CHD controls. In addition, rs7412-T and rs7259620-A were protective factors for CHD in males (rs7412-T: $\mathrm{OR}=0.527$, allele $\mathrm{P}=0.004 ;$ rs7259620-A: $\mathrm{OR}=0.743$, allele $\mathrm{P}=0.029$ ) [54]. Giannakopoulou et al. reported a sex specific increased methylation in the CTH promoter gene in 34 patients who had coronary artery bypass surgery (CABG) $(19.1 \%)$ compared to 16 control subjects $(10.3 \%)$. Increased methylation levels were observed in male CABG patients compared to male control subjects while in females this was not observed [51]. Furthermore, Xu at al., showed that CHD cases had a significantly lower methylation level of the GCK gene (mean, SD: $49.77 \pm 6.43 \%$ ) compared with controls, while there was no difference of GCK methylation level between males and females and no significant interaction between gender and disease [52]. However, a significant difference of the CpG2 methylation level with CHD was observed in males only [52]. On the other hand, one study evaluated the association between the DNA methylation profiles of genes involved in One-Carbon Metabolism (OCM) and the homocysteine (Hcy) pathway, with the myocardial infraction risk due to the low B-vitamins intake [53], based in the rationale that B-vitamins and folates pathway may modulate DNA methylation [53]. The results from this study showed an inverse association between B-vitamins intake and the hypermethylation in three genes (TCN2 promoter, CBS 50UTR, AMT genebody) in male cases, as well as two genes (PON1 gene-body, CBS 50UTR) in female cases [53].

\subsubsection{Stroke}

Four articles [15,56-58] reported sex-specific associations between DNAm and stroke. Among these, three articles [56-58] used data from the same population for their analyses. Two studies applied global and two others candidate gene approaches. These studies applied a crosssectional design, used blood samples and included a total of 1,045 nonoverlapping participants 459 women and 586 men from diverse ethnic backgrounds, such as Chinese-Taiwanese [56-58] and Spanish [15]. The summary of their sex-specific findings is shown in Supplemental Fig. 2.

Two of the studies used a candidate gene approach and performed pyrosequencing to assess methylation of the targeted regions in the gene promoter $[57,58]$, while the two other studies investigated the global DNAm of the LINE-1 elements using pyrosequencing [56] or luminometric methylation assay (LUMA) [15]. Further, two studies found a significant decrease in LINE-1 methylation in men compared to women in cases of stroke $[15,56]$. One of them also reported that cases of ischemic stroke presented a lower methylation level compared to controls. In addition, this hypomethylation of LINE-1 in men was associated with an increased stroke risk by 1.2 -fold after adjusting for risk factors, while no significant association was observed in women [56]. On the other hand, among the two studies investigating the estrogen receptor alpha $E R \alpha$ [57] and the matrix metalloproteinase-2 (MMP-2) [58] respectively, the methylation levels were lower in individuals with stroke compared to controls, in all the CpG sites analyzed in both studies. Further, when exploring the sex-specific associations, the two studies obtained contrary results. One study found a significant difference in methylation levels in all the investigated CpGs annotated to the gene ER $\alpha$ only between female cases and controls [57]. Whereas the other study reported a significant difference between the methylation levels in one out of eight CpGs annotated to MMP-2 only between male cases and controls [58]. None of these studies investigated the difference between male and female cases.

\section{Genes, pathways and cardiovascular disease}

Studies included in this systematic review report that methylated CpG sites annotated to KDM6A, PLA2G7, CETP, ABCG1, LIPC, BCL11A, ADD1, CNN1B, HOOK2, PLTP, GALNT2, PON1, TCN2, CBS, AMT, CTH, INS, GNAS-AS1, MMP2, CCDC8, MAP3K13, FTO, ESR1, CDKN2B, APOE, IGFBP7, PTPN1, GCK and PTX3 were differently methylated for men and women. An overview of these genes, function and their sex specific methylations is provided in Table 1 and Supplemental Table 2. In addition, Fig. 2 shows the prioritized pathway connectivity between cardio-metabolic genes that were found to be differentially methylated in men and women. The most significant pathways, that in Fig. 2 are shown with darker red nodes with more representative enrichment include the Vitamin B12 Metabolism, Statin Pathway, Plasma lipoprotein, Plasma lipoprotein assembly, remodeling and clearance and Cholesterol metabolism. Hence, the most important genes connecting these pathways that merit further consideration were: $A B C G 1, A P O E$, PLTP, LIPC, CETP, CTH and INS. Overall, the majority of the genes reported in this review were previously known to be associated with CVD risk factors or CVD outcomes.

\section{Discussion}

In this review, we systematically summarized the current evidence on sex differences in DNA methylation in relation to cardiometabolic diseases. We included 30 unique studies that had either stratified their analyses by sex and/or specified that their results did not differ among sexes by testing for statistical sex-interaction. Overall, our findings indicate that global DNAm might influence cardiometabolic risk in a sexspecific manner, and that DNAm at 31 gene sites could be differentially associated with various cardiometabolic traits in men and women.

\subsection{Global DNA methylation}

We identified four studies suggesting that altered LINE-1 DNAm may play a role in CVD risk in a sex-specific manner: (i) DNAm measured in LINE-1 repeats was inversely associated with different serum lipids in men and women [14,25], (ii) decreased LINE-1 was associated with higher stroke risk in the overall sample and in men [56] and (iii) DNAm measured using genomic 5-methyl cytosine content and LUMA indicated hypomethylation in male as compared to female stroke cases [15].

In the current review, global DNA hypomethylation was associated with poorer outcomes. In particular, global DNA hypomethylation was associated with higher LDL and lower HDL levels in the overall sample and in Samoan men, but not in women [14] and increased stroke risk in Chinese men but not in women ${ }^{53}$. These findings are in line with observations in healthy Caucasian men where subjects with decreased LINE-1 methylation were more likely to develop ischemic heart disease and stroke (women were not included in this study) [59]. Global DNA 
hypomethylation has been previously reported in stroke patients as compared with healthy [59] and in a large study with participants from European ancestry, decreased global DNAm was observed in male as compared to female stroke cases ${ }^{56}$. In experimental studies, global DNA hypomethylation has been shown to precede the formation of atherosclerosis in Apoe-/- mice, and has been associated with hyperhomocysteinemia and aortic lipid deposition in mutant mice deficient in methylenetetrahydrofolate reductase [60]. While in humans global loss of DNAm has been previously associated with atherosclerosis in both, atherosclerotic lesions [61] and peripheral blood leukocytes [62] but also with blood lipids, inflammation and blood pressure [10] implying that LINE-1 hypomethylation could be associated with an unfavorable cardiovascular risk profile. Global DNAm is considered a robust measurement of the overall genomic methylation which is reported to be one of the earliest molecular changes in the transition of a cell from a normal to a diseased state [63]. Blood DNA hypomethylation might be easily measured and could be used to identify people at risk of cardiovascular events. Our findings emphasize the need of sex-specific approaches when further exploring the possible role of global DNAm as a biomarker and potential intervention target in cardiometabolic diseases.

\subsection{Epigenome wide-association studies and candidate gene approach}

We identified only six studies $[12,31,41,42,44,46]$ that investigated differentially methylated regions in the genome with cardiometabolic diseases in a hypothesis-free approach. Among these, three EWAS $[12,31,44]$ reported sex-specific associations, and KDMA6A, FTO,
MAP3K13 and CCDC8 were some of the important genes that were found to be methylated in a sex-specific manner with blood lipids and glycaemia traits. Among 25 candidate gene studies, 22 studies reported sex-specific associations between DNAm and cardiometabolic diseases at the following genes PLA2G7, BCL11A, KDM6A, LIPC, ABCG1, PLTP, CETP, ADD1, CNN1B, HOOK2, GFBP-7,PTPN1, GCK, PTX3, ABCG1, GALNT2, CDKN2B, APOE, CTH, GNASAS, INS, PON1, TCN2, CBS, AMT, $M M P-2$ and $E R-\alpha$. Based on the prioritized pathway connectivity analysis, although, limited, the evidence suggests an involvement of biological pathways related to vitamin B12 metabolism, statin pathway, plasma lipoprotein, plasma lipoprotein assembly, remodeling and clearance and cholesterol metabolism, with sex differences in cardiometabolic diseases (Fig. 2). Some of the most relevant genes from the pathway analysis were ABCG1, APOE, PLTP, LIPC, CETP, CTH and INS. Overall, these genes have been associated to cardiometabolic outcomes, however little evidence links them to epigenetics and cardiometabolic diseases and even less to sex differences in cardiometabolic diseases.

$A B C G 1$ gene is a transmembrane cholesterol transporter that effluxes cellular cholesterol from macrophages by delivering cholesterol to mature high-density lipoprotein particles. Beyond a role in cellular lipid homeostasis, ABCG1 equally participates to glucose and lipid metabolism by controlling the secretion and activity of insulin and lipoprotein lipase. Moreover, there is a growing body of evidence suggesting that modulation of $A B C G 1$ expression might contribute to the development of diabetes and obesity [64], which are major risk factors of CVD. The ABCG1, GALNT2 and HMGCR genes have been previously associated with pathogenesis and progression of CHD through manipulating the various lipid pathways $[65,66]$. In the current review,

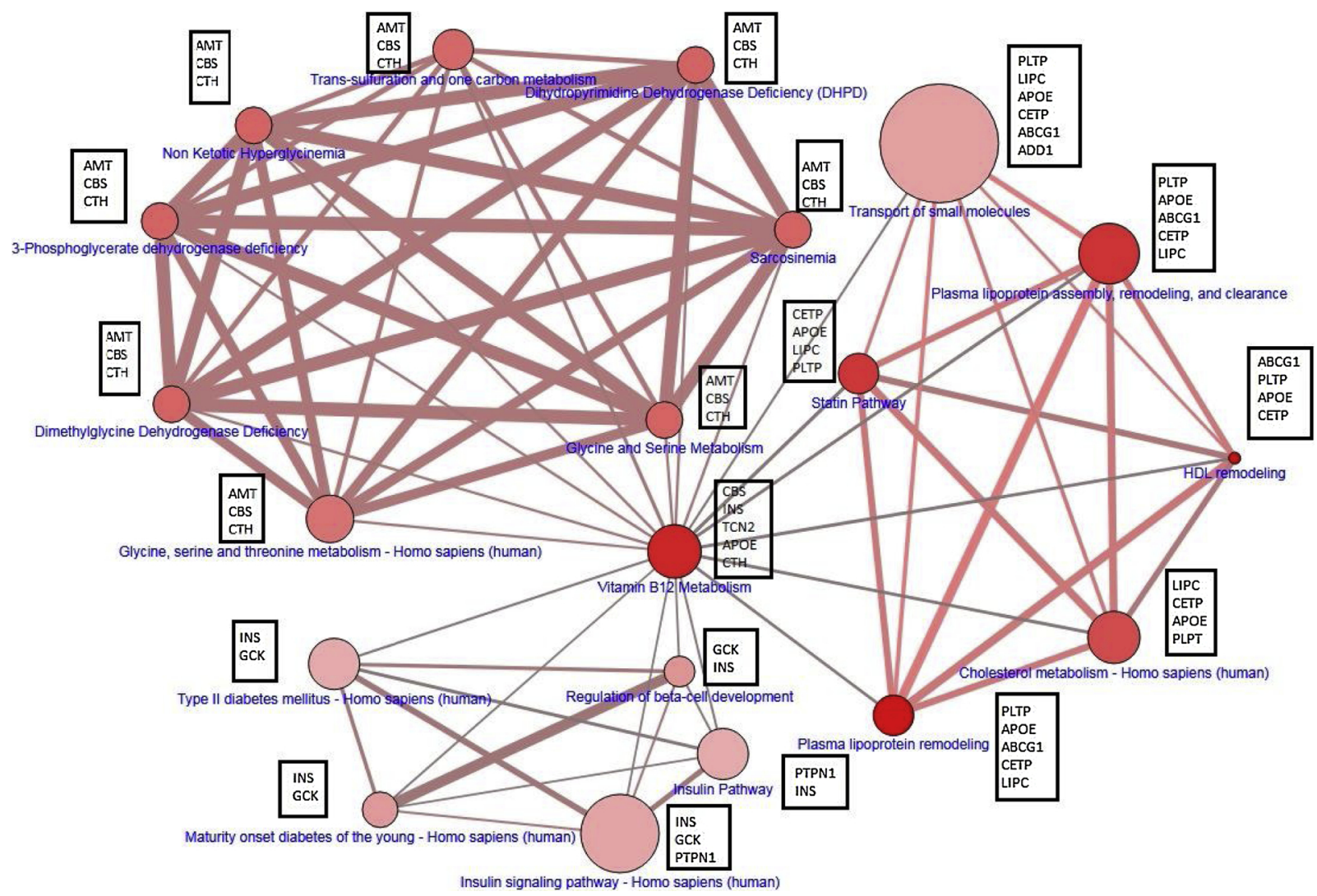

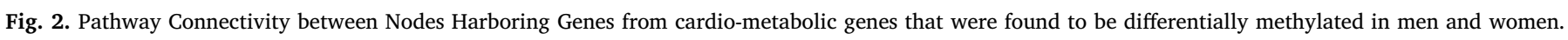

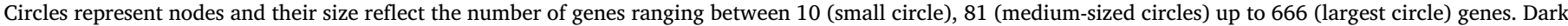

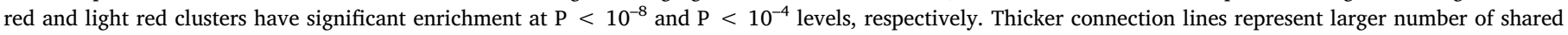

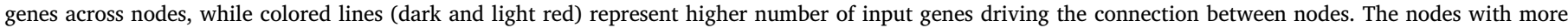

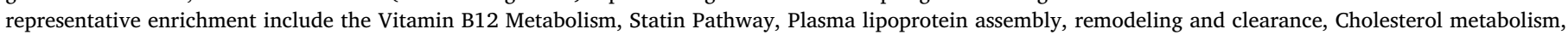

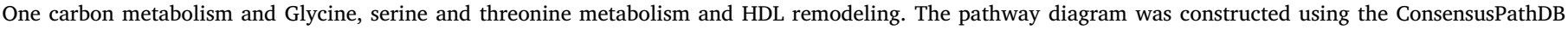
(CPDB) web-based software. 
hypermethylation of these three genes was associated with higher levels of total cholesterol and LDL, and increased CHD risk in men [50], while it was linked to higher levels of TG in women but not with risk of $\mathrm{CHD}^{7}$. The expression of $A B C G 1$ gene reduces cholesterol accumulation in macrophages by promoting the transfer of intracellular cholesterol into HDL pathway [67]. In contrast to this, hypermethylation at PLA2G7 was associated with levels of total cholesterol, triglycerides and ApoB in females but not in males, and also only female CHD cases were hypermethylated as compared to controls ${ }^{5}$. PLA2G7 is the coding gene for Lp-PLA2 whose abnormal activity can cause high risk of CHD and may serve as a diagnostic marker for CHD [68]. Therefore, the sex disparities in the ABCG1 and PLA2G7 methylation may have an effect in the molecular mechanisms underlying the sex-specific pathophysiology of CHD and may provide epigenetic clues to explain the inconsistency in the epidemiological studies. However, both studies were done in Han Chinese population, and sample size was rather small (only $85 \mathrm{CHD}$ patients and 54 participants without CHD [50] and 36 CHD cases and 36 controls). Hence, further replication studies with larger sample size and in different ethnic populations are required to confirm these findings.

The APOE gene encodes apolipoprotein $\varepsilon$ (ApoE), a protein that associates with lipids to form lipoproteins that package and traffic cholesterol and lipids through the bloodstream and has been linked with numerous physiological conditions, including healthy aging [69], cardiovascular disease [70], diabetes [71] and cognitive function [72]. One study using samples of 563 blood-bank donors, found that $1 \%$ of the inter-individual variation in plasma ApoE levels was attributable to variation of age and sex [73]. Researchers from the ApoEurope project, reported a sex-differential effect of age on mean levels of ApoE [74]. In men, the levels of ApoE levelled off after the age of 45 years, whereas they continued to increase in women [74].

PLTP (phospholipid transfer protein) is essential for the transfer of excess surface lipids from TG-rich lipoproteins to HDL particles. PLTPmediated phospholipid transport among HDL particles is also known to be associated with HDL particle size and lipid composition. Sex disparities for HDL levels associated with PLTP have been previously reported [75]. In the PAGE study, the major allele of rs7679 was associated with higher HDL levels in women only. The locus with the most consistent evidence for sex disparities across the studies is $L P L$, or lipoprotein lipase. Different SNPs in this gene exhibited sex disparities for HDL levels in two prior studies, with a larger effect in males [76,77]. In two other studies, $L P L$ exhibited sex differences for TG levels, also with a stronger effect in males $[75,78]$. LPL is the rate-limiting enzyme for hydrolysis of triglycerides in lipoproteins and polymorphisms and mutations in LPL have been associated with lipid metabolism disorders. Hormone levels have been shown to affect regulation of LPL, including thyroid hormone, estrogen, and testosterone [79], which could possibly and partially explain the observed association in cardiometabolic diseases.

Although these pathways and the respective reported genes need further investigation, confirmation and translational research, the current evidence suggests they could be biologically relevant and could hold the key for future drug discovery, diagnosis and treatment of cardiometabolic diseases overall and in a sex specific manner. Determining the relationships between genes is essential for molecular biology and medicine. These relationships often cluster together into functional and disease pathways, and the characterization of these pathways is necessary to improve disease classification, patient stratification and, ideally, personalized treatment [80].

\subsection{Epigenetic mechanisms in biological processes of sex differences}

Sex differences in pathophysiology of cardiometabolic diseases could be attributed to several gender and sex-specific factors [81]. Lifestyle factors (smoking, diet, stress) can determine gender differences by modifying cardiometabolic risk directly, and they can also modify the epigenetic marks in a sex-specific manner leading to sex differences in cardiometabolic diseases [81](Fig. 2). Sex differences may also be driven by biological dissimilarities rather than different environmental exposures among men and women [81]. In particular, the major mechanisms by which sex might influence cardiometabolic diseases epigenetics may include: (i) the genomic and non-genomic effects of steroid hormones and their receptors on DNAm enzymes, histone modifiers and miRNAs, (ii) genomic imprinting, leading to DNAm of either maternal or paternal alleles and (iii) increased expression of X-chromosomal escape genes in women targeting epigenetic modifications and the expression of non-pseudo-autosomal Y-chromosomal epigenetic modifiers in men [16].

Sex hormones have been extensively studied in the past decade in regard to cardiometabolic diseases due to the better cardiometabolic profiles in women as compared to their male counterparts. In the current review, we found some implications for the interactions between sex hormones and methylation in modifying sex differences cardiometabolic diseases. Three $[15,57,58]$ of the studies included in the current review, investigating epigenetic changes and stroke and reporting differences between men and women, highlight the importance of sex hormones and their receptors. Using a global DNA methylation approach, Soriano-Tarraga et al. [15], found that global hypomethylation was independently associated with stroke subtypes only in females. Moreover, there was an association between lower $E R \alpha$ methylation levels and large-artery and cardio-embolic stroke subtypes in women, while in men this association was not observed. It might be that women suffering from a major ischemic stroke may cause a more significant change in $E R \alpha$ methylation levels to reduce the brain injury [57]. In line with this, differential DNAm profiles mediated sex differences in the endogenous neuroprotective response to middle cerebral artery occlusion (MCAO) in mice were reported. In female mice, MACO induced selective demethylation of the ERa gene promoter, leading to the increase in ERa expression [82]. Also, sex differences were observed in MMP-2 methylation, with expression of MMP-2 being closely related to sex hormones [58]. Males with small-vessel ischemic stroke had lower methylation levels at all MMP-2 CpG sites, while no association was observed in women [58]. Further exploration of the underlying mechanisms is needed. Sex- and stroke-subtype-specific effects must be taken into consideration when investigating potential strategies to alter the activity of $M M P-2$ in patients with ischemic stroke. Steroid hormones can induce, among others, modification of histones. Androgen or estrogen receptors act by binding to hormone response elements in the DNA and attract various cofactors that have inherent histone acetyltransferase or methyl transferase activity. This is particularly known for the CREB binding protein and E1A binding protein p300 [83]. The histone-modifying enzymes alter the epigenetic state of gene promoters to which the nuclear receptors bind, thereby changing gene expression.

It is known that DNAm contributes to X-chromosome inactivation in females [84], and findings by Garcia-Calzon et al., demonstrated that DNA methylation in the X-chromosome in human liver mirrors the methylome in other human tissues [31]. They reported higher average degree of X-chromosome methylation in females than in males with 37 $\%$ of the significant sites on the X-chromosome having higher methylation in males [31]. Around $95 \%$ of the CpG sites in the X-chromosome that had differential DNAm in human liver between sexes also had different methylation levels between males and females in pancreatic islets and brain independently of the clinical characteristics of the population $[12,85]$. Further, they identified four genes on the X-chromosome with large differences in DNA methylation between males and females and being more expressed in liver from females than males: XIST, ARSE, RPS4X, and KDM6A [31]. Also, higher ARSE and RPS4X mRNA expression has been found in pancreatic islets, and higher XIST and RPS4X mRNA expression was also found in brain from females compared with males ${ }^{15}$, [85]. These differences in gene expression in several tissues may explain some metabolic differences between males and females. Interestingly, these four genes are known to escape X- 
chromosome inactivation [86-88]. In this study serum HDL levels were positively associated with KDM6A mRNA expression in human liver in addition to higher serum HDL levels and higher KDM6A expression in females. Also, silencing KDM6A in hepatocytes resulted in lower HDL levels and lower expression of key genes encoding proteins that regulate HDL levels, supporting the direct contribution of KDM6A in the differences found in HDL levels between males and females [31].

\subsection{Potential clinical implications and recommendations for future research}

Although the clinical use of epigenetic marks in the field of cardiometabolic diseases is still in its infancy this is not the case with cancer research. Molecular risk stratification studies using (epi)genetic marks have focused on identifying molecular features associated with clinical outcome and have applied them to patients' risk stratification and treatment guidance $[89,90]$. Such results indicated that a gene expression score that incorporates prognostic genetic and epigenetic information could be used as a model for treatment response but also for risk stratification and early disease detection. In particular, sex-specific epigenetic marks against or as a supplement to existing risk scores (such as the Framingham Risk Score [91]) may be an added value when predicting the risk of cardiometabolic diseases. This is also supported by our findings suggesting that for cardiometabolic traits epigenetic markers may not be equally good predictors in men and women, emphasizing the role of sex in epigenetic patterns of cardiometabolic diseases. Further, as sex is one of the strongest predictors of treatment response, the epigenetic signatures may be used as markers to indicate the successfulness of pharmacological or dietary/lifestyle interventions in cardiometabolic diseases among sexes. Given the lack of sex-stratification in studies focusing on epigenetic mechanisms and the fact that the majority of the studies were focused to epigenetic changes in autosome chromosomes in regard of cardiometabolic diseases, our review underscores the emerging need for future studies to investigate the influence of sex on epigenetic mechanisms in cardiometabolic diseases. In complex phenotypes such as cardiometabolic diseases, the collection of high-quality blood samples and metabolically active tissues could provide the basis for the creation of large data sets that should accurately incorporate the many sources of variability (age, sex/gender, race/ethnicity). In particular, future prospective observational studies should aim to explore the role of sex when studying the associations between epigenetic marks and mechanistic pathways of cardiometabolic diseases by stratifying their analyses by sex and comparing male and female participants. Second, studying the associations between DNAm and intermediate CVD risk factors is valuable, however, from the clinical perspective, the value of DNAm as a biomarker of the risk factor is as good as the intermediate risk factor itself. Therefore, as we did not identify studies focusing on outcomes such as stroke or myocardial infarction, it might be of great value for future research to investigate the role of sex on the epigenetic determinants of stroke and myocardial infarction. Third, potential biological mechanisms underlying sex-specific associations should be further explored in an experimental setting. It is now known that sex differences in morphology and in response to stress exist also in cellular levels [92-94]. Therefore, when translating the observational findings into experimental settings a clear distinction between male and female animal models or cell cultures is of high importance in order to obtain non-biased results on the sex-specific pathophysiology of cardiometabolic diseases.

\subsection{Strengths and limitations}

In this systematic review on sexually dimorphic DNAm, we critically appraised the literature following an a priori designed protocol with clearly defined inclusion and exclusion criteria using a comprehensive literature search in five databases. While previous systematic reviews on the topic are limited only to major CVD outcomes [16], our study took in consideration a broad range of cardiometabolic risk factors and diseases. However, the limitations of the findings from this study merit careful consideration. The included studies in this review were limited in sample size and the majority of studies included were cross-sectional assessments, making it difficult to conclude whether DNAm patterns are a cause or consequence of cardiometabolic changes. In addition, the results of some of the studies need cautious interpretation when it comes to the biological or functional relevance of their findings. Even though a study may report a significant difference in DNAm the biological relevance of small differences could be likely minimal and unknown. Studies investigating associations between metabolic syndrome and DNAm also need to be interpreted with caution given the heterogeneity of metabolic syndrome and that the subjects may or may not have dyslipidemia, elevated BP, and hyperglycemia. Therefore interpreting associations between changes in DNAm and subjects classified as having metabolic syndrome is Moreover, although individual studies attempted to adjust for established CVD risk factors, adjustment levels were inconsistent across the studies. Also, DNAm patterns reported in blood samples may not mirror the methylation patterns in the relevant targeted tissues. Further, we did not perform the search for non-coding microRNAs and histone modifications because the scope of our search was DNA methylation. Given the importance of microRNAs and histone modifications as epigenetic mechanisms, future systematic reviews and meta- analyses on microRNAs, histone modifications and sex differences in different types of cardiovascular tissues would be an added value on the topic. Moreover, we hand searched relevant reviews and references of studies included in the current review in order to minimize the possibility of missing important studies. Also, we cannot exclude the possibility of publication bias from underreporting negative findings. Lastly, a meaningful quantitative pooling of the existing data was unfeasible due to the heterogeneity in the input parameters, assumptions and study design.

\subsection{Conclusions}

Although a growing body of evidence suggests biological, genetic and epigenetic sex differences in cardiometabolic diseases, only a small number of studies in the field stratify or present their results by sex. Nevertheless, the cumulative evidence from the studies that reported sex-based results, suggest that epigenetic changes in specific individual genes might be differently associated with cardiometabolic traits in males and females, encouraging further and larger-scale investigation. Robust, replicable results from carefully designed studies have the potential to uncover the molecular biological processes involved in disease onset and progression. In addition, future studies should help characterize gene regulatory effects of non-coding genetic variations, and, hopefully, give indications into disease-relevant biological pathways which could be addressed by preventive or therapeutic interventions. Clearly, a considerable amount of functional work is required in the future to expand our field of view beyond the classic biological mechanisms involved in sex differences of cardiometabolic diseases, and that could be important to design new drugs that target sex-specific mechanisms and permit more precise and efficient care.

\section{Contributors}

Eralda Asllanaj contributed to literature search, acquisition, collection, interpretation of data and drafting of the manuscript.

Xiaofang Zhang contributed to literature search, acquisition, collection, interpretation of data and drafting of the manuscript.

Carolina Ochoa Rosales contributed to literature search, acquisition, collection, interpretation of data and drafting of the manuscript.

Jana Nano contributed to literature search, acquisition, collection, interpretation of data and drafting of the manuscript.

Wichor M. Bramer contributed to data search strategy.

Eliana Portilla-Fernandez contributed to literature search, acquisition, collection, interpretation of data and drafting of the manuscript. 
Kim V.E. Braun contributed to literature search, acquisition, collection, interpretation of data and drafting of the manuscript.

Valentina Gonzalez-Jaramillo contributed to literature search, acquisition, collection, interpretation of data and drafting of the manuscript.

Wolfgang Ahrens contributed to critical revision of the manuscript for important intellectual content.

Arfan Ikram contributed to contributed to critical revision of the manuscript for important intellectual content.

Mohsen Ghanbari contributed to critical revision of the manuscript for important intellectual content.

Trudy Voortman contributed to critical revision of the manuscript for important intellectual content.

Oscar H. Franco contributed to study concept and design, critical revision of the manuscript for important intellectual content.

Taulant Muka contributed to study concept and design, literature search, acquisition, collection, interpretation of data and drafting of the manuscript, and study supervision.

Marija Glisic contributed to study concept and design, literature search, acquisition, collection, interpretation of data and drafting of the manuscript, and study supervision.

\section{Conflict of interest}

The authors declare that they have no conflict of interest.

\section{Funding}

No external funding was received for the preparation of this review.

\section{Provenance and peer review}

This article has undergone peer review.

\section{Acknowledgments}

We would like to thank Maarten F.M. Engel, Sabrina Gunput and Elise Krabbendam, Biomedical Information Specialists from Erasmus MC Medical Library for help with literature search and the 24-design.com for help with the design of the figures. Carolina Ochoa-Rosales' work was kindly supported by CONICYT PAI/INDUSTRIA 72170524.

\section{Appendix A. Supplementary data}

Supplementary material related to this article can be found, in the online version, at doi:https://doi.org/10.1016/j.maturitas.2020.02. 005.

\section{References}

11] C. Manach, et al., Addressing the inter-individual variation in response to consumption of plant food bioactives: towards a better understanding of their role in healthy aging and cardiometabolic risk reduction, Mol. Nutr. Food Res. 61 (6) (2017).

[2] Y.W. Park, et al., The metabolic syndrome: prevalence and associated risk factor findings in the US population from the Third National Health and Nutrition Examination Survey, 1988-1994, Arch. Intern. Med. 163 (4) (2003) 427-436.

[3] A. Bjornerem, et al., Endogenous sex hormones in relation to age, sex, lifestyle factors, and chronic diseases in a general population: the Tromso Study, J. Clin. Endocrinol. Metab. 89 (12) (2004) 6039-6047.

[4] K.H. Humphries, et al., Sex differences in cardiovascular disease - Impact on care and outcomes, Front. Neuroendocrinol. 46 (2017) 46-70.

[5] Y. Appelman, et al., Sex differences in cardiovascular risk factors and disease prevention, Atherosclerosis 241 (1) (2015) 211-218.

[6] L.S. Mehta, et al., Acute myocardial infarction in women: a scientific statement from the american heart association, Circulation 133 (9) (2016) 916-947.

[7] D.J. Boyne, et al., Endogenous sex hormone exposure and repetitive element DNA methylation in healthy postmenopausal women, Cancer Causes Control 28 (12) (2017) 1369-1379.

[8] A.P. Feinberg, Epigenetics at the epicenter of modern medicine, JAMA 299 (11)
(2008) 1345-1350.

9] K.D. Robertson, DNA methylation and human disease, Nat. Rev. Genet. 6 (8) (2005) $597-610$.

[10] T. Muka, et al., The role of epigenetic modifications in cardiovascular disease: a systematic review, Int. J. Cardiol. 212 (2016) 174-183.

[11] K.V. Braun, et al., The role of DNA methylation in dyslipidaemia: a systematic review, Prog. Lipid Res. 64 (2016) 178-191.

[12] E. Hall, et al., Sex differences in the genome-wide DNA methylation pattern and impact on gene expression, microRNA levels and insulin secretion in human pancreatic islets, Genome Biol. 15 (12) (2014) 522.

[13] P. Yousefi, et al., Sex differences in DNA methylation assessed by $450 \mathrm{~K}$ BeadChip in newborns, BMC Genomics 16 (2015) 911.

[14] H.L. Cash, et al., Cardiovascular disease risk factors and DNA methylation at the LINE-1 repeat region in peripheral blood from Samoan Islanders, Epigenetics 6 (10) (2011) 1257-1264.

[15] C. Soriano-Tarraga, et al., Global DNA methylation of ischemic stroke subtypes, PLoS One 9 (4) (2014) e96543.

[16] R.J.G. Hartman, S.E. Huisman, H.M. den Ruijter, Sex differences in cardiovascular epigenetics-a systematic review, Biol. Sex Differ. 9 (1) (2018) 19.

[17] S. Stoll, C. Wang, H. Qiu, DNA methylation and histone modification in hyperten sion, Int. J. Mol. Sci. 19 (4) (2018).

[18] T. Muka, et al., A 24-step guide on how to design, conduct, and successfully publish a systematic review and meta-analysis in medical research, Eur. J. Epidemiol. (2019).

[19] D. Moher, et al., Preferred reporting items for systematic reviews and meta-analyses: the PRISMA statement, Int. J. Surg. 8 (5) (2010) 336-341.

[20] W.M. Bremer, Reference checking for systematic reviews using Endnote, J. Med Libr. Assoc. 106 (4) (2018) 542-546.

[21] J.E. Lewis, et al., A randomized controlled trial of the effect of dietary soy and flaxseed muffins on quality of life and hot flashes during menopause, Menopause 13 (4) (2006) 631-642.

[22] A. Kamburov, et al., The ConsensusPathDB interaction database: 2013 update, Nucleic Acids Res. 41 (Database issue) (2013) D793-800.

[23] F. Ohka, et al., The global DNA methylation surrogate LINE-1 methylation is correlated with MGMT promoter methylation and is a better prognostic factor for glioma, PLoS One 6 (8) (2011).

[24] C. Knothe, et al., Disagreement between two common biomarkers of global DNA methylation, Clin. Epigenetics (2016) 8.

[25] N. Malipatil, et al., Assessment of global long interspersed nucleotide element-1 (LINE-1) DNA methylation in a longitudinal cohort of type 2 diabetes mellitus (T2DM) individuals, Int. J. Clin. Pract. (2018) e13270.

[26] D. Jiang, et al., Elevated PLA2G7 gene promoter methylation as a gender-specific marker of aging increases the risk of coronary heart disease in females, PLoS One 8 (3) (2013).

[27] L. Tang, et al., BCL11A gene DNA methylation contributes to the risk of type 2 diabetes in males, Exp. Ther. Med. 8 (2) (2014) 459-463.

[28] S.P. Guay, et al., Epipolymorphisms within lipoprotein genes contribute independently to plasma lipid levels in familial hypercholesterolemia, Epigenetics 9 (5) (2014) 718-729.

[29] S.P. Guay, et al., DNA methylation variations at CETP and LPL gene promoter loci: new molecular biomarkers associated with blood lipid profile variability, Atherosclerosis 228 (2) (2013) 413-420.

[30] M.L. Wright, et al., Joint influence of SNPs and DNA methylation on lipids in african americans from hypertensive sibships, Biol. Res. Nurs. 20 (2) (2018) 161-167.

[31] S. Garcia-Calzon, et al., Sex differences in the Methylome and transcriptome of the human liver and circulating HDL-Cholesterol levels, J. Clin. Endocrinol. Metab. 103 (12) (2018) 4395-4408.

[32] Q. Huang, et al., Elevation of PTPN1 promoter methylation is a significant risk factor of type 2 diabetes in the Chinese population, Exp. Ther. Med. 14 (4) (2017) 2976-2982.

[33] L. Tang, et al., Elevated CpG island methylation of GCK gene predicts the risk of type 2 diabetes in Chinese males, Gene 547 (2) (2014) 329-333.

[34] L.-N. Zhang, et al., Lower ADD1 gene promoter DNA methylation increases the risk of essential hypertension, PLoS One 8 (5) (2013) e63455.

[35] Q. Zhong, et al., Association of SCNN1B promoter methylation with essential hypertension, Mol. Med. Rep. 14 (6) (2016) 5422-5428.

[36] L. Han, et al., The interactions between alcohol consumption and DNA methylation of the ADD1 gene promoter modulate essential hypertension susceptibility in a population-based, case-control study, Hypertens. Res. 38 (4) (2015) 284-290.

[37] R. Fan, et al., Association of AGTR1 promoter methylation levels with essential hypertension risk: a matched case-control study, Cytogenet. Genome Res. 147 (2-3) (2015) 95-102.

[38] S.Q. Mao, et al., Hypomethylation of interleukin-6 (IL-6) gene increases the risk of essential hypertension: a matched case-control study, J. Hum. Hypertens. 31 (8) (2017) 530-536.

[39] X.J. Bao, et al., Hypomethylation of the interferon gamma gene as a potential risk factor for essential hypertension: a case-control study, Tohoku J. Exp. Med. 244 (4) (2018) 283-290.

[40] A.E. Bostrom, et al., Longitudinal genome-wide methylation study of Roux-en-Y gastric bypass patients reveals novel CpG sites associated with essential hypertension, BMC Med. Genomics 9 (2016) 20

[41] C. Marzi, et al., Epigenetic signatures at AQP3 and SOCS3 engage in low-grade inflammation across different tissues, PLoS One 11 (11) (2016) e0166015.

[42] Y.V. Sun, et al., Gene-specific DNA methylation association with serum levels of Creactive protein in African Americans, PLoS One 8 (8) (2013) e73480.

[43] T.M. Guo, et al., Pentraxin 3 (PTX3) promoter methylation associated with PTX3 
plasma levels and neutrophil to lymphocyte ratio in coronary artery disease, J. Geriatr. Cardiol. 13 (8) (2016) 712-717.

[44] K.J. Burghardt, et al., The influence of metabolic syndrome and sex on the DNA methylome in Schizophrenia, Int. J. Genomics 2018 (2018) 8076397.

[45] S. Rodriguez-Rodero, et al., Altered intragenic DNA methylation of HOOK2 gene in adipose tissue from individuals with obesity and type 2 diabetes, PLoS One 12 (12) (2017) e0189153.

[46] G. Toperoff, et al., Genome-wide survey reveals predisposing diabetes type 2-related DNA methylation variations in human peripheral blood, Hum. Mol. Genet. 21 (2) (2012) 371-383.

[47] H.F. Gu, et al., Evaluation of IGFBP-7 DNA methylation changes and serum protein variation in Swedish subjects with and without type 2 diabetes, Clin. Epigenetics 5 (1) (2013) 20.

[48] R.P. Talens, et al., Hypermethylation at loci sensitive to the prenatal environment is associated with increased incidence of myocardial infarction, Int. J. Epidemiol. 41 (1) (2012) 106-115.

[49] D. Jiang, et al., Elevated PLA2G7 gene promoter methylation as a gender-specific marker of aging increases the risk of coronary heart disease in females, PLoS One 8 (3) (2013) e59752.

[50] P. Peng, et al., A preliminary study of the relationship between promoter methylation of the ABCG1, GALNT2 and HMGCR genes and coronary heart disease, PLoS One 9 (8) (2014) e102265.

[51] E. Giannakopoulou, et al., Epigenetics-by-Sex interaction for coronary artery disease risk conferred by the cystathionine gamma-lyase gene promoter methylation, OMICS 21 (12) (2017) 741-748.

[52] L. Xu, et al., GCK gene-body hypomethylation is associated with the risk of coronary heart disease, Biomed Res. Int. 2014 (2014) 151723.

[53] G. Fiorito, et al., B-vitamins intake, DNA-methylation of one Carbon Metabolism and homocysteine pathway genes and myocardial infarction risk: the EPICOR study, Nutr. Metab. Cardiovasc. Dis. 24 (5) (2014) 483-488.

[54] H. Ji, et al., APOE hypermethylation is significantly associated with coronary heart disease in males, Gene 689 (2019) 84-89.

[55] X. Chen, et al., Elevated methylation of cyclin dependent kinase inhibitor 2B contributes to the risk of coronary heart disease in women, Exp. Ther. Med. 17 (1) (2019) 205-213.

[56] R.T. Lin, et al., LINE-1 methylation is associated with an increased risk of ischemic stroke in men, Curr. Neurovasc. Res. 11 (1) (2014) 4-9.

[57] H.F. Lin, et al., Demethylation of circulating estrogen receptor alpha gene in cerebral ischemic stroke, PLoS One 10 (9) (2015) e0139608.

[58] H.F. Lin, et al., Methylation in the matrix metalloproteinase-2 gene is associated with cerebral ischemic stroke, J. Investig. Med. 65 (4) (2017) 794-799.

[59] A. Baccarelli, et al., Ischemic heart disease and stroke in relation to blood DNA methylation, Epidemiology 21 (6) (2010) 819-828.

[60] Z. Chen, et al., Mice deficient in methylenetetrahydrofolate reductase exhibit hyperhomocysteinemia and decreased methylation capacity, with neuropathology and aortic lipid deposition, Hum. Mol. Genet. 10 (5) (2001) 433-443.

[61] M.O. Hiltunen, et al., DNA hypomethylation and methyltransferase expression in atherosclerotic lesions, Vasc. Med. 7 (1) (2002) 5-11.

[62] R. Castro, et al., Increased homocysteine and S-adenosylhomocysteine concentrations and DNA hypomethylation in vascular disease, Clin. Chem. 49 (8) (2003) 1292-1296.

[63] T. Mikeska, J.M. Craig, DNA methylation biomarkers: cancer and beyond, Genes (Basel) 5 (3) (2014) 821-864.

[64] Z. Tavoosi, et al., Cholesterol Transporters ABCA1 and ABCG1 Gene Expression in Peripheral Blood Mononuclear Cells in Patients with Metabolic Syndrome, Cholesterol 2015 (2015) 682904.

[65] P. Jeemon, et al., Implications of discoveries from genome-wide association studies in current cardiovascular practice, World J. Cardiol. 3 (7) (2011) 230-247.

[66] I. Tietjen, et al., Segregation of LIPG, CETP, and GALNT2 mutations in Caucasian families with extremely high HDL cholesterol, PLoS One 7 (8) (2012) e37437.

[67] J.F. Oram, A.M. Vaughan, ATP-Binding cassette cholesterol transporters and cardiovascular disease, Circ. Res. 99 (10) (2006) 1031-1043.

[68] H. Grallert, et al., Eight genetic loci associated with variation in lipoprotein-associated phospholipase A2 mass and activity and coronary heart disease: meta-analysis of genome-wide association studies from five community-based studies, Eur.
Heart J. 33 (2) (2012) 238-251.

[69] N. Garatachea, et al., ApoE gene and exceptional longevity: insights from three independent cohorts, Exp. Gerontol. 53 (2014) 16-23.

[70] S. Kathiresan, et al., Polymorphisms associated with cholesterol and risk of cardiovascular events, N. Engl. J. Med. 358 (12) (2008) 1240-1249.

[71] Y.W. Yin, et al., Influence of apolipoprotein E gene polymorphism on development of type 2 diabetes mellitus in Chinese Han population: a meta-analysis of 29 studies, Metabolism 63 (4) (2014) 532-541.

[72] E. Rubino, et al., Apolipoprotein E polymorphisms in frontotemporal lobar degeneration: a meta-analysis, Alzheimers Dement. 9 (6) (2013) 706-713.

[73] E. Boerwinkle, G. Utermann, Simultaneous effects of the apolipoprotein E polymorphism on apolipoprotein E, apolipoprotein B, and cholesterol metabolism, Am. J. Hum. Genet. 42 (1) (1988) 104-112.

[74] N. Haddy, et al., The importance of plasma apolipoprotein E concentration in addition to its common polymorphism on inter-individual variation in lipid levels: results from Apo Europe, Eur. J. Hum. Genet. 10 (12) (2002) 841-850.

[75] F.W. Asselbergs, et al., Large-scale gene-centric meta-analysis across 32 studies identifies multiple lipid loci, Am. J. Hum. Genet. 91 (5) (2012) 823-838.

[76] T.M. Teslovich, et al., Biological, clinical and population relevance of 95 loci for blood lipids, Nature 466 (7307) (2010) 707-713.

[77] Y.S. Aulchenko, et al., Loci influencing lipid levels and coronary heart disease risk in 16 European population cohorts, Nat. Genet. 41 (1) (2009) 47-55.

[78] K.C. Taylor, et al., Investigation of gene-by-sex interactions for lipid traits in diverse populations from the population architecture using genomics and epidemiology study, BMC Genet. 14 (2013) 33.

[79] H. Wang, R.H. Eckel, Lipoprotein lipase: from gene to obesity, Am. J. Physiol. Endocrinol. Metab. 297 (2) (2009) E271-88.

[80] A.L. Barabasi, N. Gulbahce, J. Loscalzo, Network medicine: a network-based approach to human disease, Nat. Rev. Genet. 12 (1) (2011) 56-68.

[81] R. Jaenisch, A. Bird, Epigenetic regulation of gene expression: how the genome integrates intrinsic and environmental signals, Nat. Genet. 33 (2003) 245-254.

[82] J.M. Westberry, A.K. Prewitt, M.E. Wilson, Epigenetic regulation of the estrogen receptor alpha promoter in the cerebral cortex following ischemia in male and female rats, Neuroscience 152 (4) (2008) 982-989.

[83] J.E. Leader, et al., Epigenetic regulation of nuclear steroid receptors, Biochem. Pharmacol. 72 (11) (2006) 1589-1596.

[84] L. Carrel, H.F. Willard, X-inactivation profile reveals extensive variability in Xlinked gene expression in females, Nature 434 (7031) (2005) 400-404.

[85] H. Xu, et al., Sex-biased methylome and transcriptome in human prefrontal cortex, Hum. Mol. Genet. 23 (5) (2014) 1260-1270.

[86] C.J. Brown, et al., A gene from the region of the human X inactivation centre is expressed exclusively from the inactive X chromosome, Nature 349 (6304) (1991) 38-44.

[87] F. Lan, et al., A histone H3 lysine 27 demethylase regulates animal posterior development, Nature 449 (7163) (2007) 689-694.

[88] M.G. Lee, et al., Demethylation of H3K27 regulates polycomb recruitment and H2A ubiquitination, Science 318 (5849) (2007) 447-450.

[89] M.E. Figueroa, et al., DNA methylation signatures identify biologically distinct subtypes in acute myeloid leukemia, Cancer Cell 17 (1) (2010) 13-27.

[90] G. Marcucci, et al., Epigenetics meets genetics in acute myeloid leukemia: clinical impact of a novel seven-gene score, J. Clin. Oncol. 32 (6) (2014) 548-556.

[91] S.G. Wannamethee, et al., Metabolic syndrome vs Framingham Risk Score for prediction of coronary heart disease, stroke, and type 2 diabetes mellitus, Arch. Intern. Med. 165 (22) (2005) 2644-2650.

[92] R. Addis, et al., Human umbilical endothelial cells (HUVECs) have a sex: characterisation of the phenotype of male and female cells, Biol. Sex Differ. 5 (1) (2014) 18 .

[93] M. Lorenz, K.J. Kaufmann, Kreye K, Mertens C, Kuebler M, Baumann G. WM, G. Gossing, A. Marki, A. Zakrzewicz, C. Miéville, A. Benn, D. Horbelt, P.R. Wratil, K. Stangl, V. Stangl, Does cellular sex matter? Dimorphic transcriptional differences between female and male endothelial cells, Atherosclerosis 240 (1) (2015) 61-72.

[94] L. Du, H.R, H. Bayir, S.C. Watkins, V.A. Tyurin, F. Guo, P.M. Kochanek, L.W. Jenkins, J. Ren, G. Gibson, C.T. Chu, V.E. Kagan, R.S. Clark, Starving neurons show sex difference in autophagy, J. Biol. Chem. 284 (4) (2009) 2383-2396. 